\title{
The theory and simulation of relativistic electron beam transport in the ion-focused regime
}

\author{
Stephen B. Swanekamp \\ Naval Research Laboratory, Plasma Physics Division, Washington, DC 20375-5000 \\ James Paul Holloway, Terry Kammash, and Ronald M. Gilgenbach \\ Department of Nuclear Engineering, University of Michigan, Ann Arbor, Michigan 48109-2104
}

(Received 28 August 1991; accepted 17 January 1992)

\begin{abstract}
Several recent experiments involving relativistic electron beam (REB) transport in plasma channels show two density regimes for efficient transport; a low-density regime known as the ion-focused regime (IFR) and a high-pressure regime. The results obtained in this paper use three separate models to explain the dependency of REB transport efficiency on the plasma density in the IFR. Conditions for efficient beam transport are determined by examining equilibrium solutions of the Vlasov-Maxwell equations under conditions relevant to IFR transport. The dynamic force balance required for efficient IFR transport is studied using the particle-in-cell (PIC) method. These simulations provide new insight into the transient beam front physics as well as the dynamic approach to IFR equilibrium. Nonlinear solutions to the beam envelope are constructed to explain oscillations in the beam envelope observed in the PIC simulations but not contained in the Vlasov equilibrium analysis. A test particle analysis is also developed as a method to visualize equilibrium solutions of the Vlasov equation. This not only provides further insight into the transport mechanism but also illustrates the connections between the three theories used to describe IFR transport. Separately these models provide valuable information about transverse beam confinement; together they provide a clear physical understanding of REB transport in the IFR.
\end{abstract}

\section{INTRODUCTION}

Charged particle beams generated from high-power diodes always have velocity components perpendicular to the direction of propagation. This motion originates from sources such as imperfections in the diode shaping fields, electromagnetic forces from the beam self-fields, scattering through thin foils, or random thermal motions. Transverse motions from all these sources act to increase the beam radius, which may be detrimental in applications that require the beam transport over large distances with little or no radial expansion. One technique to control transverse beam motion uses the focusing properties of large electromagnets. However, magnetic lenses can be difficult and expensive for high-energy electrons or ions since the power required in these electromagnets increases with beam energy.

An alternative method of suppressing transverse motion in electron beams using the strong electrostatic focusing properties of an ion channel was first suggested by Bennett. ${ }^{1,2}$ In this focusing method the repulsive electric field of the electron beam is reduced by a positive ion channel, which allows the beam to self-focus in its own magnetic field. One of the earliest experimental demonstrations of ion channel guided electron beams are those of Graybill and Nablo. ${ }^{3}$ These experiments injected a $3.6 \mathrm{MV}, 17 \mathrm{kA}, 20$ nsec electron beam pulse into neutral gas at various pressures. Figure 1 shows the transported beam energy density as a function of gas pressure measured by Graybill and Nablo $20 \mathrm{~cm}$ downstream from the injection point. The two large peaks in Fig. 1 show that efficient beam propagation depends critically on the background gas pressure. These peaks represent the transport of over $80 \%$ of the total beam energy, indicating that ion channel guiding of a relativistic electron beams has the potential to be an efficient method of transport.

A qualitative explanation of the dependence of transport efficiency on pressure is given below. As the beam electrons move through the gas, electron-ion pairs are produced as a result of ionizing collisions by the primary beam. These ions provide the positive space charge necessary to partially neutralize the space charge of the beam. At very low gas pressures the ionization produced by impact ionization is not sufficient to radially confine the beam and the beam rapidly expands resulting in a low transport efficiency. As gas pressure increases, the number of ions in the channel becomes sufficient to provide radial confinement and transport efficiency increases. However, eventually more ionization is

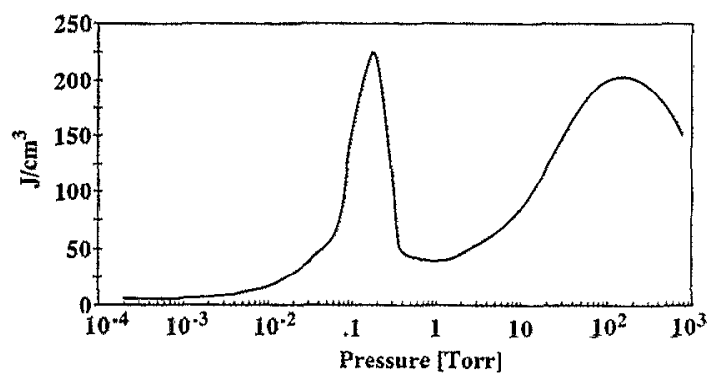

FIG. 1. Transported electron beam energy density on axis $20 \mathrm{~cm}$ downstream from the injection point (after Graybill and Nablo ${ }^{\dagger}$ ). 
produced than is needed to space charge neutralize the beam and a significant number of plasma electrons are trapped in the channel by the excess positive charge. These extra electrons can interact resonantly with the primary beam electrons and lead to a two-stream instability that disrupts efficient beam transport. At higher gas pressures growth of the two-stream instability is collisionally damped and the transport efficiency again increases. At very high gas pressures the beam's space charge is completely neutralized and, since the channel conductivity is large, the trapped channel electrons react to the longitudinal emf induced by the rising beam current to produce a sizable return current. Magnetic interactions between these oppositely directed current streams can cause transverse oscillations in the beam-plasma channel, which eventually interrupts efficient beam transport. This mechanism of instability is known as the resistive hose instability and is responsible for the decrease in efficiency at very high pressures. In this paper we present a detailed analysis of the low-pressure ion-focused regime (IFR) propagation window. Research at the Naval Research Laboratory (NRL) over the past several years has been primarily concerned with relativistic electron beam transport in the high-pressure regime. ${ }^{4,5}$ However, recent experiments at NRL have shown the utility of IFR cells to precondition the emittance variation in the beam for stabilation of the resistive hose instability. ${ }^{6}$

The low-pressure propagation window associated with the first peak in Fig. 1 is known as the ion-focused regime (IFR); it is defined by gas pressures that are high enough to provide a sufficient number of ions to allow self-focused electron transport yet low enough that excess channel electrons are not produced in sufficient numbers to drive an instability. To maintain strict control over the channel conductivity it is critical that the plasma electrons not produce excessive amounts of ionization as they leave the channel. Qualitatively, this condition can be met if the mean-free path for cascade ionization is much larger than the beam radius. ${ }^{7.8}$ Therefore, to avoid avalanche breakdown in the channel, the background gas pressure should be kept low. On the other hand, the gas pressure must be high enough for the beam to quickly create the ionization necessary to achieve self-pinched IFR transport. One way around this apparent difficulty is to keep the gas pressure low and use a laser or secondary low-energy electron beam to preionize the plasma channel in a very lowpressure gas $\left(p \sim 10^{-3}\right.$ Torr $)$. This provides the necessary charges to achieve self-pinched transport while avoiding beam-induced ionizations and avalanche breakdown. Another advantage of preionized channels is that they provide a sufficiently high plasma density inside the channel while having a low plasma density outside the channel. This ensures that there is a strongly preferred direction of propagation and takes full advantage of the focusing properties of the plasma channel. In addition to electron produced ionizations, it has been suggested that ion-induced ionizations and resonant charge exchange could also play a role in IFR transport expcriments. ${ }^{9}$ Thesc effects are ignored in the present work. Therefore the results obtained in this paper are limited to situations where gas pressures are low enough to avoid significant amounts of ion-induced ion production or where ion energies are below several hundred $\mathrm{keV}$ so that the cross section for ion-induced ionizations is low.

Since the work of Graybill and Nablo a number of researchers have studied IFR electron transport. A summary of recent IFR transport experiments is given in Table I. In this table the column $v / \gamma$ is a measure of the beam intensity, where $v=N_{b} e^{2} / m_{e} c^{2}$ is Budker's parameter, ${ }^{10} N_{b}$ is the number of beam electrons per axial length, $m_{e}$ is the electron mass, $e$ is the magnitude of the electron charge, $c$ is the speed of light, and $\gamma$ is the relativistic mass factor of the beam. As this table shows, ion channel focusing of a relativistic electron beam has been successful over a wide range of beam parameters. Like the work of Graybill and Nablo many of

TABLE I. Summary of recent IFR experiments.

\begin{tabular}{|c|c|c|c|c|c|c|c|}
\hline Author & $\begin{array}{l}\text { Lab and } \\
\text { Year }\end{array}$ & $\begin{array}{l}\text { Voltage } \\
\text { (MV) }\end{array}$ & $\begin{array}{l}\text { Current } \\
\text { (kA) }\end{array}$ & $\begin{array}{l}\text { Pulse } \\
\text { length (nsec) }\end{array}$ & $v / \gamma$ & $\begin{array}{l}\text { Length } \\
\text { (m) }\end{array}$ & $\begin{array}{l}\text { Pressure/Gas } \\
\text { (Torr) }\end{array}$ \\
\hline \multirow{6}{*}{$\begin{array}{l}\text { Miller and } \\
\text { Gilgenbach } \\
\text { Lucey and } \\
\text { Gilgenbach } \\
{\text { O'Brien } \text { et } a l^{18}}^{18}\end{array}$} & Michigan & \multirow[b]{2}{*}{0.33} & \multirow[b]{2}{*}{0.2} & 400 & 0.009 & \multirow[b]{2}{*}{1.20} & $10^{-4}-10^{-5}$ \\
\hline & 1988 & & & & & & $\mathrm{Ar}, \mathrm{He}, \mathrm{Ne}$ \\
\hline & Michigan & \multirow[b]{2}{*}{0.7} & \multirow[b]{2}{*}{0.3} & 600 & 0.008 & \multirow[b]{2}{*}{1.20} & $10^{-3}-10^{-4}$ \\
\hline & 1987 & & & & & & Diethylaniline \\
\hline & SNL & \multirow[b]{2}{*}{2.2} & \multirow[b]{2}{*}{1.2} & 900 & 0.015 & \multirow[b]{2}{*}{21.0} & $3 \times 10^{-5}$ \\
\hline & 1987 & & & & & & $\mathrm{Ar}, \mathrm{He}, \mathrm{Xe}$ \\
\hline \multirow[t]{2}{*}{ Carlson et al..$^{16}$} & LANL & \multirow[b]{2}{*}{30} & \multirow[t]{2}{*}{0.75} & 5 & 0.001 & \multirow{2}{*}{$\begin{array}{l}3.50 \\
13.0\end{array}$} & $10^{-3}$ \\
\hline & 1986 & & & & & & Benzene \\
\hline \multirow[t]{2}{*}{ Shope et al. ${ }^{15}$} & SNL & 1.0 & 14.0 & 50 & 0.300 & \multirow{2}{*}{$\begin{array}{l}1.0 \\
1.3\end{array}$} & $10^{-3}-10^{-4}$ \\
\hline & 1986 & 3.6 & 25.0 & & 0.180 & & Diethylaniline \\
\hline \multirow[t]{2}{*}{ Smith et al. ${ }^{14}$} & NSWC & \multirow[b]{2}{*}{0.7} & \multirow{2}{*}{4.0} & 100 & 0.110 & \multirow[b]{2}{*}{1.0} & $0.02-2.4$ \\
\hline & 1986 & & & & & & $\mathrm{Ar}, \mathrm{He}, \mathrm{Air}$ \\
\hline \multirow[t]{2}{*}{ Martin et al. ${ }^{13}$} & LLNL & & \multirow[b]{2}{*}{8.0} & 25 & 0.048 & \multirow[b]{2}{*}{4.0} & $10^{-5}$ \\
\hline & 1985 & 4.5 & & & & & $\mathrm{Ar}, \mathrm{He}, \mathrm{Air}$ \\
\hline \multirow[t]{2}{*}{ Wilson ${ }^{12}$} & NBS & & & 2000 & 0.020 & \multirow[b]{2}{*}{4.5} & $10^{-4}-5.0$ \\
\hline & 1981 & 0.75 & 0.75 & & & & $\mathrm{He}, \mathrm{Air}$ \\
\hline \multirow[t]{2}{*}{ Briggs et al." } & LLNL & 5.0 & 0.4 & 300 & 0.002 & \multirow[b]{2}{*}{7.0} & $0.1-200$ \\
\hline & 1977 & 1.5 & 15.0 & 30 & 0.230 & & Ar, $\mathrm{He}, \mathrm{Air}$ \\
\hline \multirow{2}{*}{$\begin{array}{l}\text { Graybill and } \\
\text { Nablo" }\end{array}$} & IPC & \multirow{2}{*}{3.6} & \multirow{2}{*}{17.0} & 20 & 0.170 & \multirow{2}{*}{0.2} & $10^{-4}-200$ \\
\hline & 1966 & & & & & & $\mathrm{Ar}, \mathrm{He}, \mathrm{Air}$ \\
\hline
\end{tabular}


the early IFR experiments relied on beam impact ionization to create the plasma channels. ${ }^{11,12}$ However, many of the recent IFR experiments have used preionized plasma channels created by either a laser-initiated discharge ${ }^{13-17}$ or a secondary low-energy electron beam. ${ }^{18,19}$

There are other effects that can influence the efficiency of IFR transport, for example, electron-ion instabilities. ${ }^{20}$ The main electron-ion instabilities relevant to IFR transport experiments are the electron-ion two-stream and ionhose instabilities. These instabilities play a crucial role in IFR transport experiments since they persist even when the channel is stable to electron-electron interactions. The time scales associated with electron-ion interactions effectively limits the pulse length of the beam that can be reliably transported using ion channels. This paper does not treat such interactions but will consider the unstable interactions that occur between beam and plasma electrons.

In this paper the application of three models: VlasovMaxwell theory, particle-in-cell simulations (PIC), and the beam envelope approach are used to analyze IFR transport. In Sec. II, self-pinched beam transport is studied from the framework of the relativistic Vlasov-Maxwell equations. The main purpose of Sec. II is to review the previous kinetic results and set the theoretical foundation for the remainder of the paper. Since similar calculations to those presented in Sec. II can be found elsewhere, only the main results are emphasized. ${ }^{21}$ Additional discussions of the conditions necessary for efficient transport are developed and estimates for beam radius are provided. These results are shown to be insensitive to the specific functional form chosen for the distribution function and therefore of practical utility.

There has been relatively few published papers featuring PIC simulations IFR electron transport. The two-dimensional simulations presented by Olson ${ }^{9}$ et al. neglected beam pressure and were performed with only one background density. The simulations performed by Hubbard et al. were mainly concerned with the degradation of beam quality as the beam passes through a transition region between IFR and solenoidal transport. ${ }^{22}$ The simulations performed by Joyce et al . concentrated on the large-amplitude plasma oscillations about the neutralization radius caused by the beam's wake fields. ${ }^{23}$ Three-dimensional simulations have been recently performed to study phenomena such as the ion-hose instability ${ }^{24}$ and IFR transport around $180^{\circ}$ bends in the recirculating linear accelerator. ${ }^{25}$ In Sec. III of this paper, two-dimensional PIC simulations of pinched beam transport are presented for several different background plasma densities and the dynamic approach to equilibrium is studied. These simulations include effects from beam pressure and provide confirmation of the Vlasov equilibrium results given in Sec. II. In addition, the qualitative aspects of the PIC results confirm the experimentally measured dependence of IFR transport efficiency on beam pressure (Fig. 1).

Several new developments are made in the envelope analysis presented in Sec. IV. First an estimate of emittance growth from multiple small-angle scatterings off of neutral gas atoms is developed. This analysis shows that neutral gas scattering can be ignored in low-pressure IFR discharges, but becomes more important as gas pressure is increased. In contrast to the self-similar treatment of Lee and Cooper, ${ }^{26}$ we opt for a treatment in which ions are stationary. This approximation provides better agreement between the envelope analysis and the PIC treatment. This is largely due to the fact that ions do not move much over the time scale of the simulations. Another major contribution of the paper is the development of a method for constructing exact solutions to the envelope equation for situations where energy losses are small, no external focusing magnets are present, and emittance growth from gas scattering is negligible. This new approach not only produces a more general result but also provides additional insight into the nature of the envelope oscillations observed in the simulations.

Since beams ultimately consist of a large number of individual electrons, a test particle approach is also developed in Sec. IV. This not only provides a convenient way to visualize solutions to the Vlasov equation but, as seen in that section, a few streams of test particles provide a detailed physical picture of the dynamic force balance that must be maintained to achieve good beam confinement. Furthermore, test particle theory illustrates the connections between the three different theories used in this paper to describe IFR transport. In the final section of the paper the important conclusions from this work are summarized.

\section{EQUILIBRIUM VLASOV ANALYSIS OF PINCHED BEAM TRANSPORT}

A schematic of the geometry used in this paper to study IFR transport is given in Fig. 2. As this figure shows, IFR transport is studied in a cylindrical polar coordinate system with the $z$ axis along the axis of symmetry, $r$ the radial distance from the $z$ axis, and $\theta$ the azimuthal angle. Beam transport is assumed to occur inside a conducting cylindrical drift tube of radius $R_{w}$ and axial length $L$. The beam is assumed to he cylindrically symmetric with a radius of $r_{b}$. It is further assumed that a cylindrically symmetric preionized plasma channel with a radius comparable to $r_{b}$ is located along the axis of the drift tube. Some of the main assumptions used to simplify the equilibrium analysis of IFR transport are the following.

(1) Collisions between beam electrons and the plasma chantiel are ignored as well as collisions between the beam and the neutral gas.

(2) Equilibrium quantities are assumed to be functions of $r$ only.

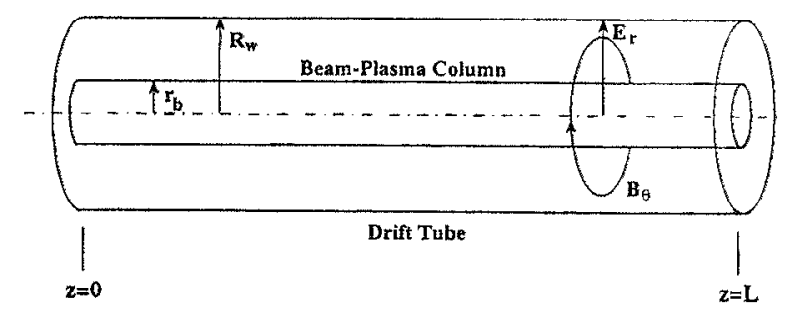

FIG. 2. Schematic of the beam-plasma channel illustrating the basic geometry. 
(3) The ratio $v / \gamma$ is assumed to be small compared to unity. When this condition is satisfied, transverse beam motions are small compared to the axial motion so that the paraxial approximation is valid $\left(\beta_{1} \ll \beta_{z}\right)$. In this case the relativistic mass factor of the beam can be written as

$$
\gamma=\left(1-\beta_{1}^{2}-\beta_{z}^{2}\right)^{-1 / 2} \cong\left(1-\beta_{z}^{2}\right)^{-1 / 2},
$$

where $\beta_{1}$ and $\beta_{z}$ are the transverse and axial velocity components normalized to the speed of light. From Table $I$ it is seen that the assumption $v / \gamma \ll 1$ is consistent with most of the IFR experiments and is not considered too restrictive.

(4) Ionization produced by the beam or channel electrons is assumed to be negligible so that the number of electron-ion pairs is constant (i.e., beam-induced ionizations and avalanche breakdown are assumed to be negligible).

(5) The mean azimuthal velocity of the various channel components is assumed to be small so that there are no net currents in the $\theta$ direction.

(6) For simplicity, it is assumed that the plasma ion and electron density profiles have the same radial dependence as the electron beam density. In this case the plasma ion and electron density will be written as

$$
\begin{aligned}
& n_{b o}(r)=f_{i} n_{b 0}(r), \\
& n_{e 0}(r)=f_{e} n_{b 0}(r),
\end{aligned}
$$

where $n_{b o}(r)$ is the beam electron density, $f_{i}$ is the charge neutralization fraction, and $f_{\mathrm{e}}$ is a measure of the number of trapped plasma electrons. In addition, in the equilibrium analysis it is assumed that ions are stationary and the plasma electron current density profile is assumed to be proportional to the electron beam current density profile and given by

$$
J_{e 0}(r)=-f_{m} e n_{b 0}(r) V_{b z}(r),
$$

where $f_{m}$ is the current neutralization fraction and $V_{b z}(r)$ is the beam's axial velocity profile.

In the absence of external fields, the steady-state Vlasov equation for the relativistic electron beam can be written as

$$
\mathbf{v} \cdot \frac{\partial f_{b 0}}{\partial \mathbf{x}}-e\left(\mathbf{E}_{0}+\frac{\mathbf{v} \times \mathbf{B}_{0}}{c}\right) \cdot \frac{\partial f_{b 0}}{\partial \mathbf{p}}=0,
$$

where $f_{b 0}(\mathbf{x}, \mathbf{p})$ is the distribution of beam particles in phase space ( $\mathbf{x}, \mathbf{p})$, and $\mathbf{E}_{0}$ and $\mathbf{B}_{0}$ are the equilibrium self-electric and magnetic fields. From assumption (2) about the equilibrium geometry it is possible to define potentials $\Phi(r)$ and $A_{z}(r)$ such that

$$
\mathbf{E}_{0}=E_{r o}(r) \mathbf{e}_{\mathbf{r}}=-\frac{\partial \Phi}{\partial r} \mathbf{e}_{\mathbf{r}}
$$

and

$$
\mathbf{B}_{0}=B_{\theta 0}(r) \mathbf{e}_{\theta}=-\frac{\partial A_{z}}{\partial r} \mathbf{e}_{\theta} .
$$

Using these relations in Gauss' and Ampère's laws, equations for $\Phi(r)$ and $A_{z}(r)$ can be written as

$$
\begin{aligned}
& \frac{1}{r} \frac{\partial}{\partial r} r \frac{\partial \Phi}{\partial r}=-4 \pi e n_{b 0}(r)\left(f_{i}-f_{e}-1\right), \\
& \frac{1}{r} \frac{\partial}{\partial r} r \frac{\partial A_{z}}{\partial r}=\frac{4 \pi}{c} e n_{b 0}(r) V_{b z}(r)\left(1-f_{m}\right) .
\end{aligned}
$$

The boundary conditions used for the solution of Eqs. (7) and (8) are that $\Phi$ and $A_{z}$ as well as $d \Phi / d r$ and $d A_{z} / d r$ be zero at $r=0$. Since $f_{e}$ must be positive and $f_{e}=f_{i}-1$ to maintain charge neutrality, Eq. (7) shows that no plasma electrons can be confined in the channel when $f_{i} \leqslant 1$. Therefore, when the plasma density exceeds the beam density, the excess ions in the channel trap plasma electrons, which can lead to unstable interactions between the trapped plasma electrons and the beam electrons. Since the purpose of the equilibrium analysis is to study IFR transport in regimes that are stable to electron-electron instabilities, it is assumed that $f_{i} \leqslant 1$ so that no plasma electrons are trapped. Therefore both $f_{e}$ and $f_{m}$ are set to zero in the following analysis.

It is well known that an arbitrary function of the single particle constants of the motion is a solution to the steadystate Vlasov equation. ${ }^{27}$ Thus the problem of finding Vlasov equilibria can be reduced to finding the single particle constants of the motion in the equilibrium fields. For beam equilibria that are azimuthally symmetric and independent of the axial coordinate $z$, the single particle constants of the motion are the energy $H$, the canonical angular momentum $P_{\theta}$, and canonical axial momentum $P_{z}$, which are given by

$$
\begin{aligned}
& H=\left(\mathbf{p}^{2} c^{2}+m_{e}^{2} c^{4}\right)^{1 / 2}-e \Phi(r), \\
& P_{\theta}=r p_{\theta}, \\
& P_{z}=p_{z}-(e / c) A_{z}(r) .
\end{aligned}
$$

There is considerable freedom in choosing the functional form of the distribution function. In this paper it is assumed that all the beam electrons have the same canonical axial momentum and the distribution function is taken to be independent of the canonical angular momentum. Under these assumptions, solutions to the steady-state Vlasov equalion can be written in the form

$$
f_{b 0}(\mathbf{x}, \mathbf{p})=F_{b 0}(H) \delta\left(P_{z}-P_{0}\right),
$$

where $P_{0}$ is the common canonical axial momentum and $F_{b 0}(H)$ is the distribution in total energy. For the moment the distribution in total energy is left arbitrary and some general properties of Eq. (10) are explored.

Once the distribution function is specified, many interesting properties of the equilibrium can be studied. Of interest in Eqs. (7) and (8) are the equilibrium density and fluid velocity profiles, which are

$$
\begin{aligned}
n_{b 0}(r) \equiv & \int_{-\infty}^{\infty} d p_{r} \int_{-\infty}^{\infty} d p_{\theta} F_{b 0}(H), \\
\mathbf{V}_{b}(r) \equiv & \frac{1}{n_{b 0}(r)} \int_{-\infty}^{\infty} d p_{r} \int_{-\infty}^{\infty} d p_{\theta} \int_{-\infty}^{\infty} d p_{z} \mathbf{v} \\
& \times F_{b 0}(H) \delta\left(P_{z}-P_{0}\right),
\end{aligned}
$$

where the components of velocity are specified in terms of the momentum by $\mathbf{v}=\mathbf{p} / \gamma m_{e}$ and $\gamma=\left(1+\mathbf{p}^{2} / m_{e}^{2} c^{2}\right)^{1 / 2}$. For the subclass of distribution functions given in Eq. (10) it can be shown that the radial and azimuthal fluid velocities are zero. This follows directly from Eq. (12) since $H$ is an even function of $p_{r}$ and $p_{\theta}$ so that $v_{r} F_{b 0}$ and $v_{\theta} F_{b 0}$ are odd and the transverse momentum integrals vanish. This property is very important for self-focused electron transport since there is no net radial flux through any cylindrical surface for any choice of $F_{b 0}(H)$. The mean axial velocity can be found 
by performing the integrations in Eq. (12) and expressed as

$$
V_{b z}(r)=p_{z}(r) / \gamma_{z}(r) m_{e}
$$

where $p_{z}(r)=P_{0}+e A_{z}(r) / c$ is the beam's axial momentum and the paraxial approximation has been used to write

$$
\gamma_{z}(r) m_{e} c^{2}=\left[p_{z}^{2}(r) c^{2}+m_{e}^{2} c^{4}\right]^{1 / 2} .
$$

The characteristic beam energy is defined by evaluating Eq. (14) at $r=0$, and is given by

$$
\gamma_{0} m_{e} c^{2}=\left(P_{0}^{2} c^{2}+m_{e}^{2} c^{4}\right)^{1 / 2} .
$$

Equations (13) and (14) show that, when $P_{0} \gg e A_{z} / c$, the beam's axial momentum profile is approximately uniform $\left[p_{z}(r) \cong P_{0}\right]$ and $\gamma_{z}(r) \cong \gamma_{0}$. But $P_{0} \gg e A_{z} / c$ is equivalent to the condition $v / \gamma \ll 1$. Therefore, within the context of small $v / \gamma$, distribution functions of the form given by Eq. (10) have axial velocity profiles that are approximately uniform.

In addition to the density and mean fluid velocity, a third macroscopic variable of interest in IFR experiments is the transverse temperature profile defined by

$$
T_{\lrcorner b}(r) \equiv \frac{1}{n_{b 0}(r)} \int_{0}^{\infty} d p_{r} \int_{0}^{\infty} d p_{\theta} \frac{p_{r}^{2}+p_{\theta}^{2}}{2 \gamma_{z}(r) m_{e}} F_{b 0}(H) .
$$

In the remainder of this section the density and transverse temperature profiles will be examined for two choices of $F_{b 0}(H)$.

As a first example, consider a beam in which all the electrons have the same total energy with a distribution in $H$, given by

$$
F_{b 0}(H)=\frac{\hat{n}_{b 0}}{2 \pi \gamma_{0} m_{e}} \delta\left(H-\gamma^{*} m_{e} c^{2}\right),
$$

where $\gamma^{*} m_{e} c^{2}$ is the common beam electron energy chosen so that $\gamma^{*}>\gamma_{0}$. This restriction on $\gamma^{*}$ is necessary since it will be shown that $\left(\gamma^{*}-\gamma_{0}\right) m_{e} c^{2}$ is related to the transverse temperature on axis, which is inherently positive. Consistent with the assumption that the transverse beam momentum is small compared to the axial momentum, the paraxial approximation can be used to simplify the expression for the total energy to yield

$$
H \cong \gamma_{z}(r) m_{e} c^{2}+\frac{p_{r}^{2}+p_{\theta}^{2}}{2 \gamma_{z}(r) m_{e}}-e \Phi(r),
$$

where $\gamma_{z}(r)$ is defined in Eq. (14). Substituting the assumed distribution in $H$ given in Eq. (27) into Eq. (11) and using the approximation for $H$ given in Eq. (18) the beam density can be expressed as ${ }^{28}$

$n_{b a}(r)=\hat{n}_{b 0} \frac{\gamma_{z}(r)}{\gamma_{0}} \begin{cases}1, & \frac{r^{2} \omega_{\beta}^{2}(r)}{c^{2}} \leqslant\left(\frac{2 T_{\perp 0}}{\gamma_{0} m_{e} c^{2}}\right), \\ 0, & \frac{r^{2} \omega_{\beta}^{2}(r)}{c^{2}}>\left(\frac{2 T_{\perp 0}}{\gamma_{0} m_{e} c^{2}}\right),\end{cases}$

where $T_{10}=\left(\gamma^{*}-\gamma_{0}\right) m_{e} c^{2}$ and

$$
\omega_{B}^{2}(r)=\frac{2 c^{2}}{r^{2}}\left(\frac{\gamma_{z}(r)-\gamma_{0}}{\gamma_{0}}-\frac{e \Phi(r)}{\gamma_{0} m_{e} c^{2}}\right)
$$

is the generalized betatron frequency. A Taylor expansion of Eq. (20) about $r=0$ shows that

$$
\omega_{\beta}^{2}(r)=\Omega_{\beta}^{2}+O\left(r^{2}\right),
$$

where the constant

$$
\Omega_{\beta}^{2}=\frac{\omega_{p b}^{2}}{2 \gamma_{0}}\left(f_{i}-\frac{1}{\gamma_{0}^{2}}\right)
$$

is the betatron frequency and $\omega_{p t}^{2}=4 \pi \hat{n}_{b 0} e^{2} / m_{e}$ is the plasma frequency of the beam electrons. It is important to note, when the beam density profile is uniform, that all the higherorder terms in the expansion are zero so that $\omega_{\beta}^{2}(r)=\Omega_{\beta}^{2}$.

Since $\gamma_{z}(r)$ and $\omega_{\beta}^{2}(r)$ depend on the potentials $A_{z}(r)$ and $\Phi(r)$, which, in turn, depend on $n_{b 0}(r)$, Eq. (19) represents an implicit expression for the density. However, when $v / \gamma \ll 1$ it can be shown that $\gamma_{z}(r) \approx \gamma_{0}$ so that the beam density is approximately uniform and $\omega_{\beta}^{2}(r) \approx \Omega_{\beta}^{2}$. In this case the beam density profile becomes

$$
n_{b 0}(r) \approx \begin{cases}\hat{n}_{b 0}, & r \leqslant r_{b}, \\ 0, & r>r_{b},\end{cases}
$$

where $r_{b}$ is the characteristic beam radius defined by

$$
r_{b}^{2}=\left(\frac{2 T_{10}}{\gamma_{0} m_{e} c^{2}}\right) \frac{c^{2}}{\Omega_{\beta}^{2}} .
$$

Equation (23) shows that, within the context of small $v / \gamma$, a uniform beam density profile with a sharp radial boundary at $r_{b}$ satisfies the equilibrium Vlasov equation. It will be shown in Sec. IV that this sharp boundary is the envelope of turning points in the radial oscillations of individual beam particles.

Substituting the form for the distribution assumed in Eq. (17) into Eq. (16) and using the approximation for $H$ given in Eq. (18) to perform the integration the transverse temperature profile can be expressed as

$$
T_{1 b}(r)=T_{10}\left[1-\frac{r^{2}}{r_{b}^{2}}\left(\frac{\omega_{\beta}^{2}(r)}{\Omega_{\beta}^{2}}\right)\right] .
$$

From Eq. (25) it is apparent that the constant $T_{\perp 0}=\left(\gamma^{*}-\gamma_{0}\right) m_{e} c^{2}$ is the beam's transverse temperature at $r=0$. In the limit of small $\nu / \gamma, \omega_{\beta}^{z}(r) \approx \Omega_{\beta}^{2}$ and the transverse temperature profile can be approximated by

$$
T_{1, b}(r) \cong T_{10}\left(1-r^{2} / r_{b}^{2}\right)
$$

Therefore, in the limit of small $v / \gamma$, the distribution given by Eq. (17) is characterized by a uniform density profile with a parabolic temperature profile with the beam's thermal energy concentrated near the axis and dropping off to zero at $r=r_{b}$.

Equation (24) gives the beam radius in terms of the beam's transverse temperature and the equilibrium selffields through the betatron frequency, $\Omega_{\beta}^{2}$. Since $r_{b}^{2}$ is necessarily positive, Eq. (24) and the definition of $\Omega_{\beta}^{2}$ [Eq. (22)] lead to the conclusion that $f_{i}$ must exceed $1 / \gamma_{0}^{2}$ to radially confine the beam in the channel. However, as $f_{i} \rightarrow 1 / \gamma_{0}^{2}$ the betatron frequency approaches zero and $r_{b} \rightarrow \infty$. The physical origins of this behavior can be understood by recognizing that when $f_{i} \approx 1 / \gamma_{0}^{2}$ the net force on the beam from the self-electric and magnetic fields is small and the beam expands as a result of its transverse kinetic pressure. Therefore, to have a tight beam pinch, there must also be a sufficient 
number of ions in the channel to balance beam expansion caused by the transverse kinetic pressure of the beam. For a beam with a given radius, transverse temperature, and current a convenient way to express this condition is

$$
\begin{aligned}
f_{i} & =\frac{1}{\gamma_{0}^{2}}+\left(\frac{T_{10}}{\gamma_{0} m_{e} c^{2}}\right)\left(\frac{4 \gamma_{0} c^{2}}{\omega_{p b}^{2} r_{b}^{2}}\right) \\
& =\frac{1}{\gamma_{0}^{2}}+\left(\frac{T_{10}}{\gamma_{0} m_{e} c^{2}}\right) \frac{I_{\mathrm{A}}}{I_{b}},
\end{aligned}
$$

where $I_{b}=\pi r_{b}^{2} e \hat{n}_{b 0} c \beta_{b z}$ is the beam current and $I_{\mathrm{A}}=\left(m_{i} c^{3} / e\right) \gamma_{0} \beta_{b z}$ is the Alfvén current. ${ }^{29}$ The first term in Eq. (27) arises from the beam's self-electric and magnetic fields while the second term is a result of its transverse pressure. To avoid unstable electron-electron interactions that can disrupt efficient beam transport an additional restriction on the charge neutralization is that $f_{i}$ must be smaller than unity. This implies that, for a beam to be both radially confined and stable to electron-electron instabilities, the charge neutralization fraction predicted by Eq. (27) must be less than unity.

As a second example consider a beam distribution in total energy $H$, which is given by

$F_{b 0}(H)=\frac{\hat{n}_{b 0}}{2 \pi \gamma_{0} m_{e} T_{\perp 0}} \exp \left(-\frac{\left(H-\gamma_{0} m_{c} c^{2}\right)}{T_{\perp 0}}\right)$.

Substituting this expression into Eq. (11) and using the approximation for $H$ given in Eq. (18) the beam density profile can be expressed as

$$
n_{b 0}(r)=\hat{n}_{b 0} \frac{\gamma_{z}(r)}{\gamma_{0}} \operatorname{cxp}\left[-\frac{r^{2}}{r_{b}^{2}}\left(\frac{\omega_{\beta}^{2}(r)}{\Omega_{\beta}^{2}}\right)\right],
$$

where the characteristic beam radius, $r_{b}$, is defined by Eq. (24), and $\omega_{\beta}^{2}(r)$ is defined by Eq. (20). Substituting the Taylor expansion of $\omega_{\beta}^{2}(r)$ [Eq. (21)] into Eq. (29) and retaining only the first term, the beam density profile near the origin can be expressed as

$$
n_{b 0}(r)=\hat{n}_{b 0} \exp \left(-r^{2} / r_{b}^{2}\right) .
$$

Since Eq. (30) is based on a Taylor expansion it is only valid for $r \ll r_{b}$. A more accurate solution for the density can be obtained by solving Eq. (29) iteratively using Eq. (30) as an initial guess for $n_{b 0}(r)$. After several iterations, this procedure converges to the Bennett distribution, which is given by

$$
n_{b 0}(r)=\hat{n}_{b 0}\left(\frac{1}{1+r^{2} / a_{b}^{2}}\right)^{2}
$$

where $a_{b}=\sqrt{2} r_{b}$ is the characteristic Bennett radius. The density profile for a beam-plasma channel with parameters $r_{b}=1.3 \mathrm{~cm}, \hat{n}_{b 0}=6.65 \times 10^{10} \mathrm{~cm}^{-3},\left(\gamma_{0}-1\right) m_{e} c^{2}=5$ $\mathrm{MeV}$, and $f_{i}=1$ is shown in Fig. 3. As this figure shows, the density profile is approximately Gaussian out to $r \approx r_{b}$, but past this point the beam density falls off more slowly and is more diffuse. The radial dependence of $\omega_{\beta}^{2}(r) / \Omega_{\beta}^{2}$ is shown in Fig. 4. As this figure shows, particles at different radii oscillate with different frequencies. This is known as phase mixing and is responsible for many interesting phenomena in nonuniform beam profiles.

Substituting the assumed form for the distribution given

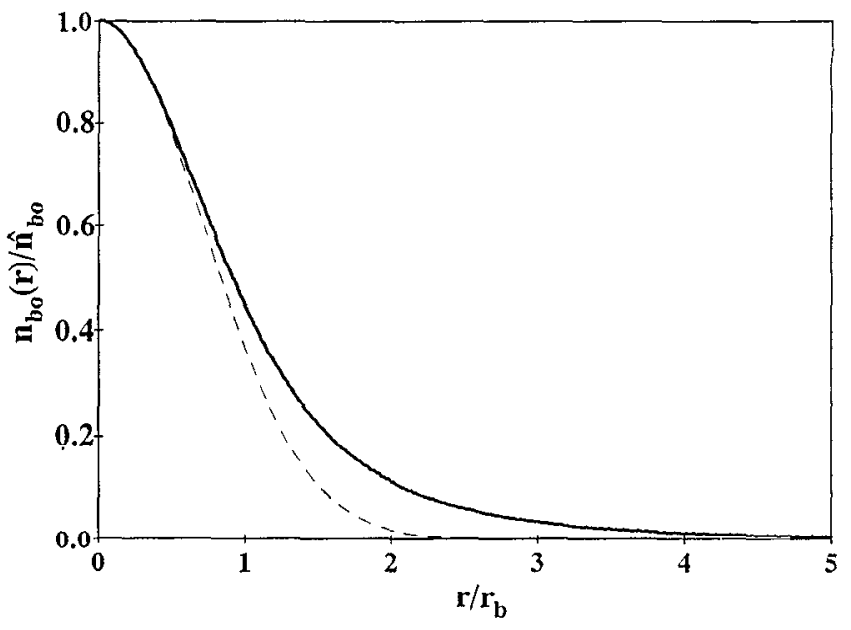

FIG. 3. Comparison of the exact density profile (solid line) obtained iteratively with that of a Gaussian profile (dashed line) for a beam-plasma channel with parameters $r_{b}=1.3 \mathrm{~cm}, \hat{n}_{b 0}=6.65 \times 10^{10} \mathrm{~cm}^{-3}$, $\left(\gamma_{0}-1\right) m_{e} c^{2}=5 \mathrm{MeV}$, and $f_{i}=1.0$.

in Eq. (28) into Eq. (16) and using the approximation for $H$ given in Eq. (18), the beam temperature profile can be expressed as

$$
T_{\perp b}(r)=T_{10} .
$$

Therefore the choice of $F_{b 0}$ given by Eq. (28) leads to a beam equilibrium characterized by a Bennett density profile and an isothermal transverse temperature profile. It can be shown that, for the Bennett distribution, equilibrium between the transverse pressure and focusing from the ion channel is maintained when

$$
\frac{a_{b}^{2}}{2}=\left(\frac{2 T_{10}}{\gamma_{0} m_{e} c^{2}}\right) \frac{c^{2}}{\Omega_{\beta}^{2}} .
$$

Equation (33) is identical to Eq. (24) so that the number of ions needed to provide beam confinement given by Eq. (27)

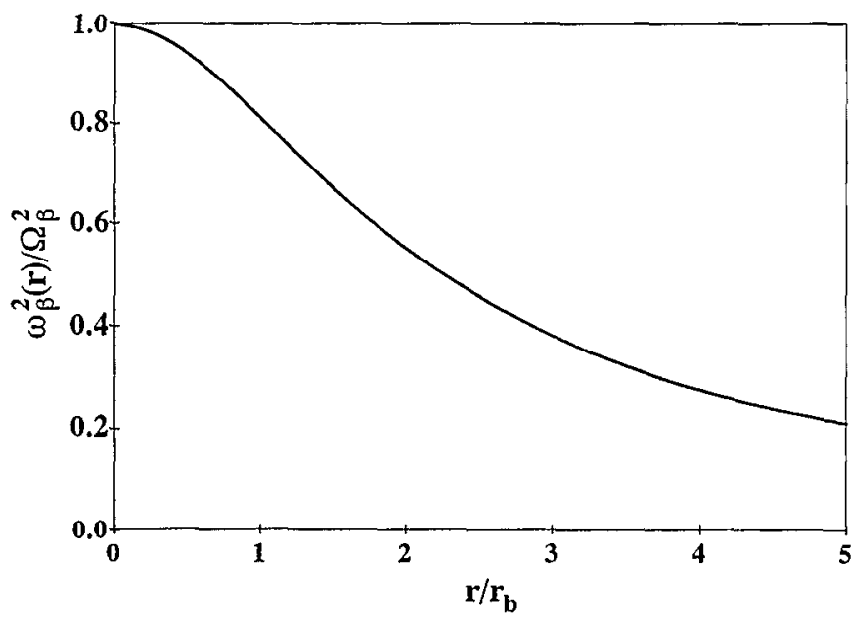

FIG. 4. The radial dependence of the generalized betatron frequency, $\omega_{\beta}^{2}(r)$, for a beam-plasma channel with parameters $r_{b}=1.3 \mathrm{~cm}$, $\hat{n}_{b 0}=6.65 \times 10^{10} \mathrm{~cm}^{-3},\left(\gamma_{0}-1\right) m_{e} c^{2}=5 \mathrm{MeV}$, and $f_{t}=1.0$. 
also holds for the Bennett profile.

The analysis presented in this section shows that radial force balance is achieved when the focusing provided positive ion channel is sufficient to balance radial expansion caused by beam self-fields and transverse pressure. For a desired beam radius, current, and transverse temperature, the number of ions required for radial force balance is given by Eq. (27). This condition is shown to be insensitive to the choice of $F_{b 0}$. Estimates of the beam radius vary between $r_{b}$ for the uniform beam profile to $\sqrt{2} r_{b}$ for the Bennett profile, where $r_{b}$ is given by Eq. (24). In the next section the dynamics of IFR transport near equilibria such as those just developed are studied using the PIC method.

\section{PIC SIMULATIONS OF IFR TRANSPORT}

To gain a deeper understanding of IFR transport, twodimensional $(r, z)$ PIC simulations of relativistic electron beam transport in preionized plasma channcls were performed using the MAGIC code, which was developed by Mission Research Corp. ${ }^{30}$ The simulations are performed in the cylindrical geometry depicted in Fig. 2 with a perfectly conducting drift tube of radius $3.5 \mathrm{~cm}$ and length $1.0 \mathrm{~m}$. To simulate the presence of thin foils, each end of the simulation is terminated by a perfectly conducting boundary. To initiate a simulation, a neutral argon plasma is loaded along the axis of the drift tube, beam electrons are launched from $z=0$, and the subsequent bchavior of the system is monitored. Because of the long axial dimension, plots of various quantities along the $z$ direction are presented in pairs of figures; one for the range $0<z<0.5 \mathrm{~m}$ and a second for the range $0.5<z<1.0 \mathrm{~m}$. Snapshots of various properties of the beam-plasma channel are shown at $t=3.3 \mathrm{nsec}$ after injection so that the beam front has had just enough time to propagate the length of the drift tube. This choice provides a view of the transient beam front physics as well as the beam's approach to equilibrium. In some cases the simulations were carried out to $6 \mathrm{nsec}$, but aside from the transient physics at the beam front, the results were not significantly different than the results presented in this section. Although ions are permitted to move in the simulations, the ions do not move appreciably over the time scale of the simulations. Therefore, no information related to ion motion is included in this paper. This certainly does not imply that ion motion is unimportant on a longer time scale.

The initial distribution of beam electrons is chosen by sampling the distribution function given by Eq. (17) which, as shown in the previous section, is characterized by a uniform density and parabolic transverse temperature profiles. The beam current is ramped up from zero to $1.7 \mathrm{kA}$ in $1 \mathrm{nsec}$ and the initial beam kinetic energy was taken to be $5.0 \mathrm{MeV}$. The desired beam radius and transverse beam temperature on axis are chosen to be $1.3 \mathrm{~cm}$ and $50 \mathrm{keV}$, respectively. Prior to beam injection a uniform charge neutral plasma channel was created along the axis of the drift tube with channel radius comparable to the beam radius; this plasma was taken to be initially at rest. From Eq. (27) the charge neutralization fraction required for efficient transport of a beam with these parameters is $f_{i} \approx 0.98$. The simulations presented in this section are done with three different plasma densities corresponding to charge neutralization fractions of $f_{i}=0.1, f_{i}=1.0$, and $f_{i}=6.0$. The case with $f_{i}=1.0$ is chosen to represent efficient IFR transport, whereas the $f_{i}=0.1$ and $f_{i}=6.0$ cases are chosen to correspond to points on the left and right of the peak IFR transport efficiency shown in Fig. 1.

The first simulation results are those representing efficient IFR transport with $f_{i}=1.0$. The positions of the beam and plasma electrons at $t=3.3 \mathrm{nsec}$ are shown in Figs. 5 and 6 , respectively. In these figures a dot is drawn to represent the location of a particle in the system. However, since the volume element varies like $r^{2}$, particle density cannot easily be inferred from the density of dots in these figures. Figure 5 (a) shows the beam electron positions in the range $0.5<z<1.0 \mathrm{~m}$. The most striking feature of this figure is a considerable amount of radial expansion and erosion of the beam front. A detailed quantitative description of the physics associated with the expansion and erosion of the beam front is very complex. ${ }^{18,31} \mathrm{~A}$ qualitative understanding of the erosion process can be gained by considering a small axial slice at the front of the beam. Since it takes a finite amount of time for plasma electrons to be accelerated from the channel, the focusing force provided by the ions is small at the beam head and the first beam slice rapidly expands due
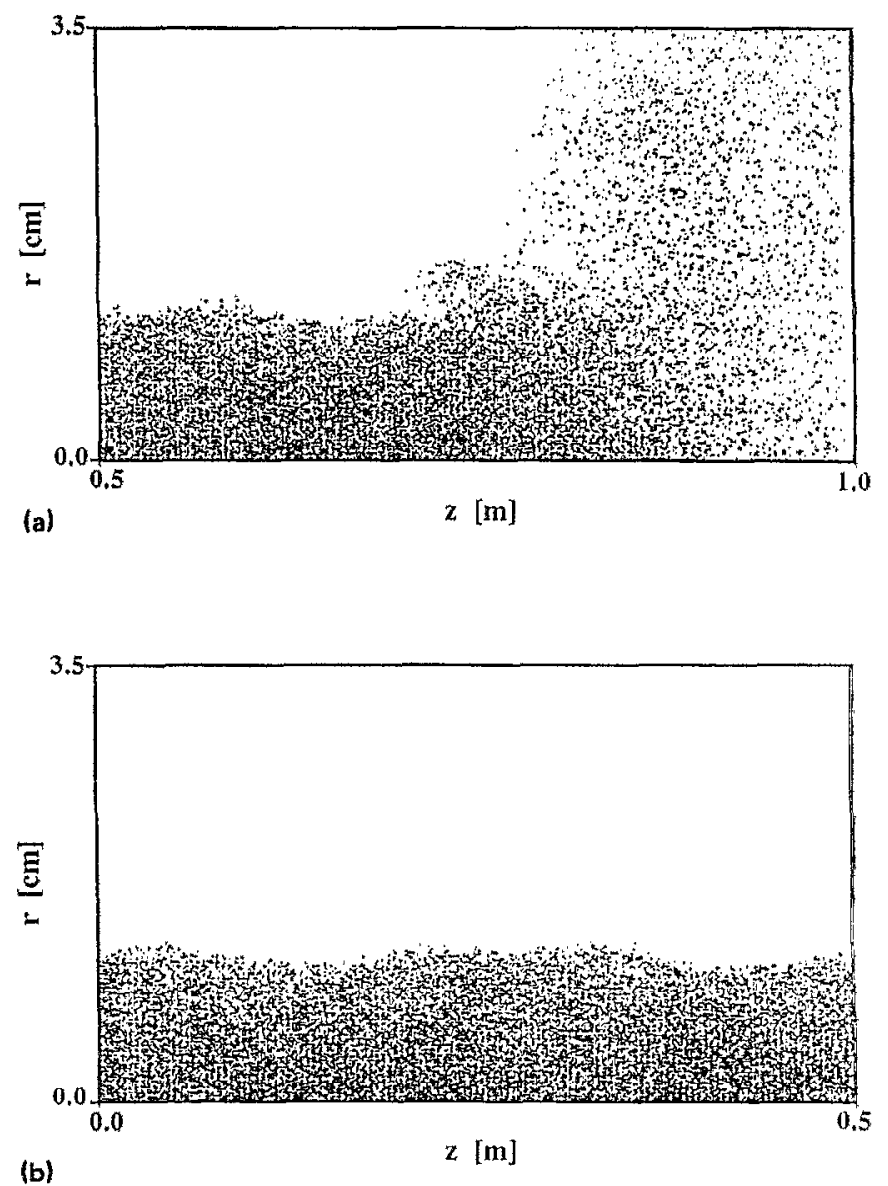

FIG. 5. Beam electron positions at $t=3.3 \mathrm{nsec}$ for an initial plasma prefill corresponding to $f_{t}=1.0$. (a) $0.5<z<1.0(\mathrm{~m})$. (b) $0<z<0.5(\mathrm{~m})$. 

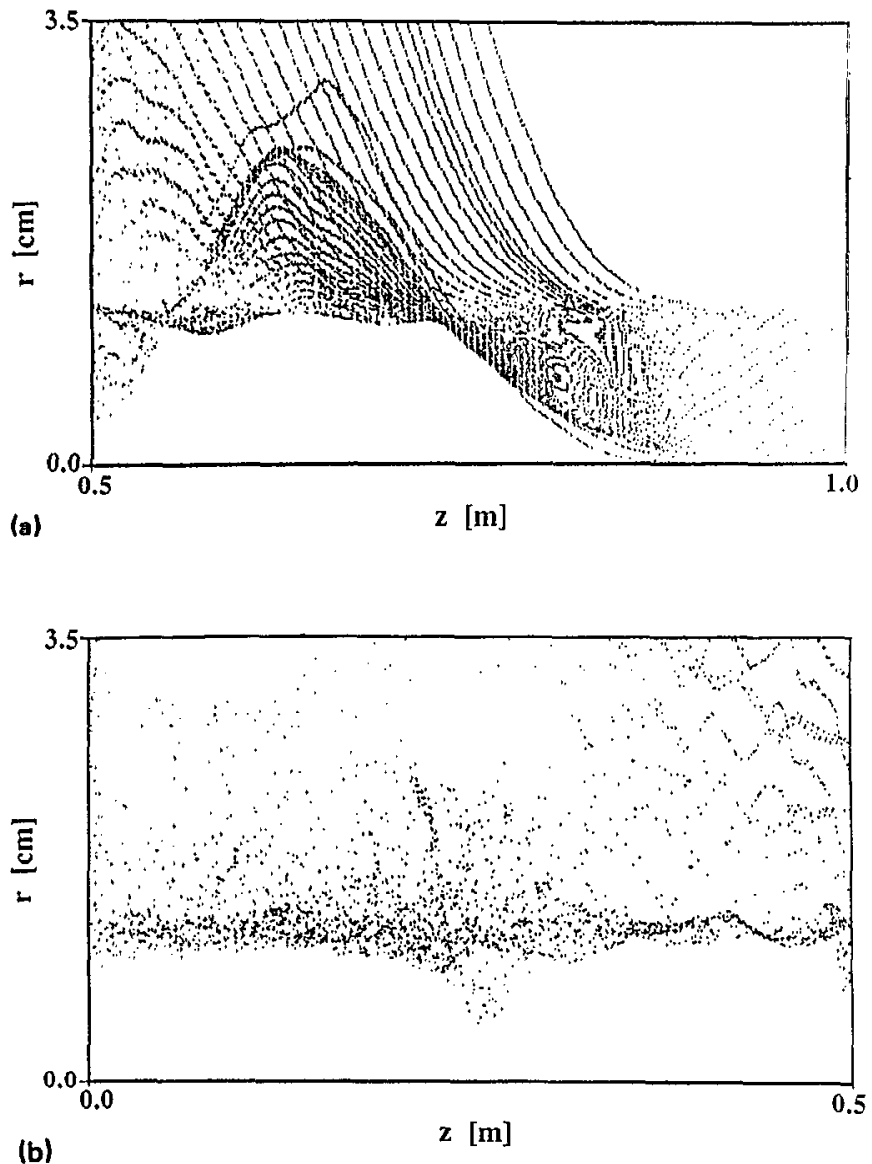

FIG. 6. Plasma electron positions at $t=3.3 \mathrm{nsec}$ for an initial plasma prefill corresponding to $f_{1}=1.0$. (a) $0.5<z<1.0$ (m). (b) $0<z<0.5(\mathrm{~m})$.

to its kinetic pressure. While the beam head is expanding, the self-electric field of the beam provides a radial acceleration of the plasma electrons toward the drift tube wall. However, this radial electric field is greatly reduced as the beam slice expands and eventually the field becomes too small to effectively accelerate any more plasma electrons from the channel. Subsequent beam slices then encounter stronger yet still very small transverse focusing forces and suffer the same fate while expelling yet more plasma electrons from the channel. Eventually, at a certain distance behind the beam front, enough plasma electrons are forced out of the channel and the beam electrons become trapped in the ion channel. The process by which electrons at the beam front are continually expended to create the ion channel for the remaining portion of the beam is termed erosion. Since erosion of the beam front constantly occurs during IFR transport it can be a significant source of electron loss, especially in IFR transport experiments of short beam pulses over very long distances.

It is seen from the positions of the beam and plasma electrons in the range $0.5<z<1.0 \mathrm{~m}$ at $t=3.3 \mathrm{nsec}$ shown in Figs. 5(a) and 6(a) that once the plasma electrons are accelerated out of the channel a tight beam pinch occurs. Since $f_{i}=1.0$ in these simulations the beam's self-electric field is neutralized so that the tight beam pinch is maintained by achieving a balance between the magnetic pressure associat- ed with the beam's self-magnetic field and the transverse kinetic pressure. The radius of the beam pinch is about 1.3 $\mathrm{cm}$, which agrees well with the equilibrium value given in Eq. (24). Figure $6(\mathrm{~b})$ shows that a small fraction of the plasma electrons remain in the simulation just outside the beam channel. These remaining electrons had very little effect on beam transport during the time scale of the simulations.

The axial momenta (actually $\gamma v_{z}$ ) of the beam electrons at $t=3.3 \mathrm{nsec}$ are shown in Fig. 7. The dip in the beam's axial momentum in the range $0.5<z<1.0 \mathrm{~m}$ can be attributed to the energy loss experienced by the beam electrons in the creation of the ion channel. This loss of beam energy appears as a corresponding gain of kinetic energy by the plasma electrons as they are expelled from the channel. Once plasma electrons are expelled from the channel no further energy is lost by the beam and the axial momentum approaches the value at injection.

The radial momenta (actually $\gamma v_{r}$ ) of the beam electrons at $t=3.3 \mathrm{nsec}$ are shown in Fig. 8. The spread in the radial momentum of the beam in the pinched beam region is consistent with a $50 \mathrm{keV}$ transverse temperature. Near the beam front it is seen that the transverse momentum spread is very narrow and the average radial momentum is positive. This occurs because those electrons with the largest trans-

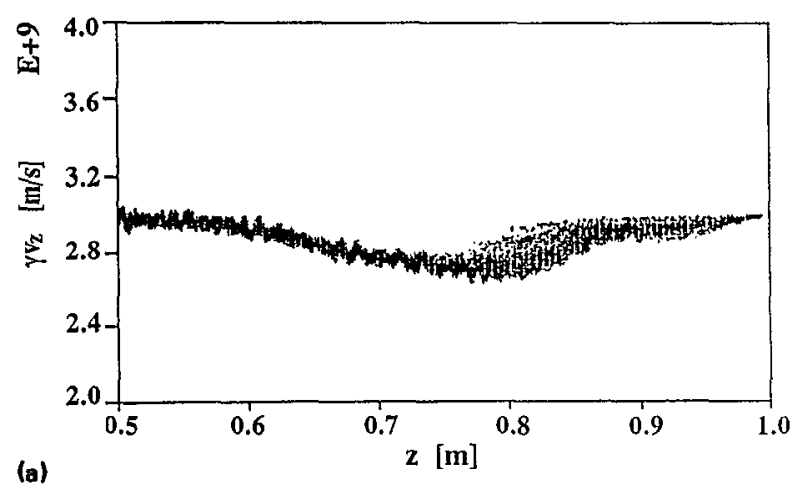

(a)

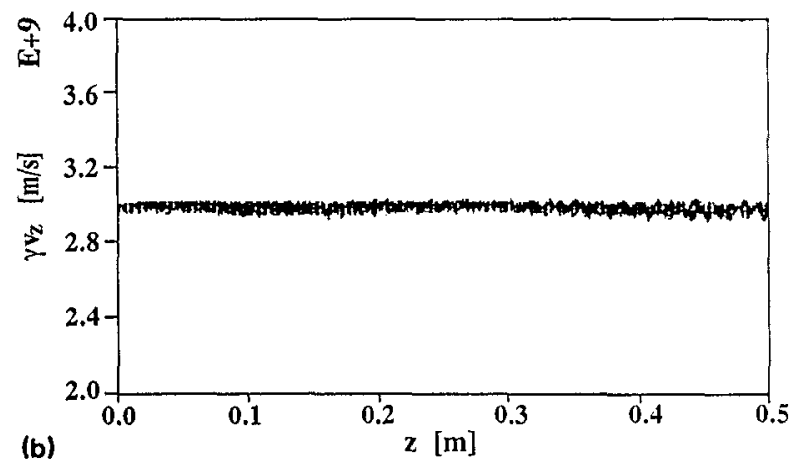

FIG. 7. Axial momentum $\left(\gamma v_{z}\right)$ at $t=3.3 \mathrm{nsec}$ for the beam electrons for an initial plasma prefill corresponding to $f_{i}=1.0$. (a) $0.5<z<1.0(\mathrm{~m})$. (b) $0<z<0.5(\mathrm{~m})$. 


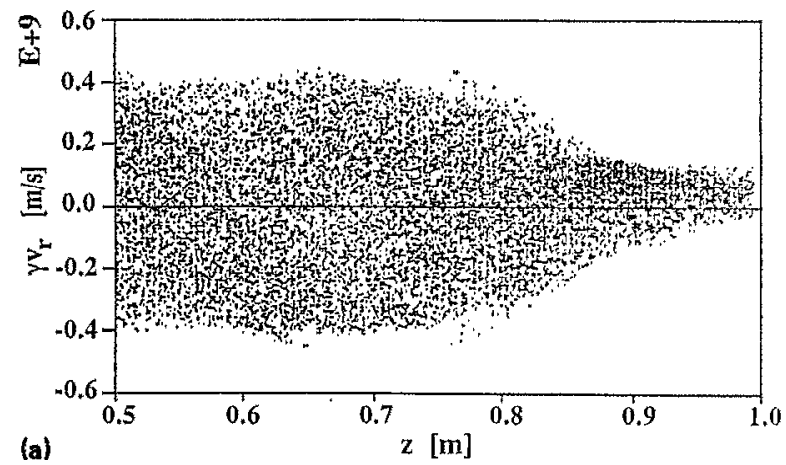

(a) $\mathrm{z}[\mathrm{m}]$

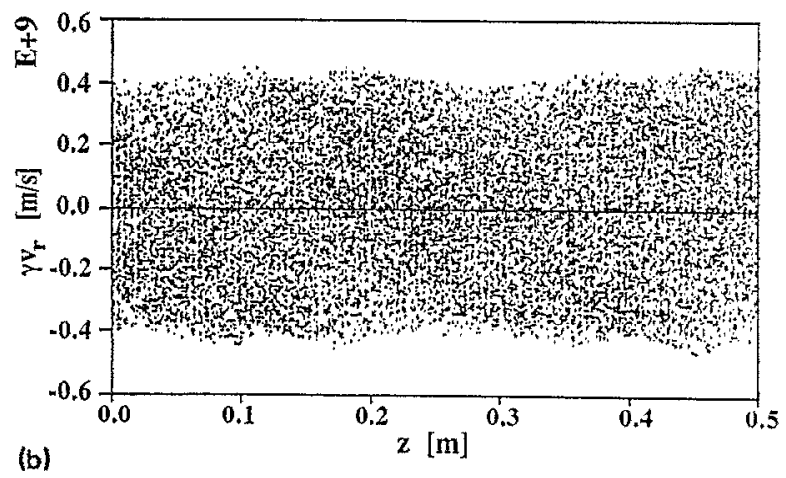

FIG. 8. Radial momentum $\left(\gamma v_{r}\right)$ at $t=3.3 \mathrm{nsec}$ for the beam electrons for an initial plasma prefill corresponding to $f_{f}=1.0$. (a) $0.5<z<1.0(\mathrm{~m})$. (b) $0<z<0.5(\mathrm{~m})$.

verse velocities are the first to be lost at the drift tube wall when focusing from the ion channel is small.

A second simulation representing efficient transport with $f_{i}=1.0$ was done with the injected beam radius no longer well matched to the equilibrium radius predicted by Eq. (24). In this case, the initial beam radius and transverse temperature were chosen so that the beam emittance was the same as in the previous case. The beam emittance $\epsilon$ is given by

$$
\epsilon=r_{\text {inj }}\left(T_{10} / 2 \gamma_{0} m_{e} c^{2}\right)^{1 / 2},
$$

where $r_{\text {inj }}$ is the beam injection radius. For the parameters used in these simulations the beam emittance is approximately $88 \mathrm{~cm}$ mrad. The injection radius for this simulation was chosen to be $1.0 \mathrm{~cm}$ so that, to have constant emittance, the initial transverse temperature of the beam was increased to $85 \mathrm{keV}$. Positions of the beam electrons at $t=3.3 \mathrm{nsec}$ for the case of a mismatched injection radius are shown in Fig. 9. A comparison of Figs. 5 and 9 shows that the transient behavior of the beam electrons is very similar. The most notable difference between these figures is the oscillation in the beam envelope apparent for the mismatched injection case. The wavelength of the envelope oscillation is estimated to be $28 \mathrm{~cm}$, which is roughly half the betatron wavelength $\left(\lambda_{\beta}=2 \pi c / \Omega_{\beta}\right)$. In the next section it will be shown that the oscillation of the beam envelope is produced by imbalances
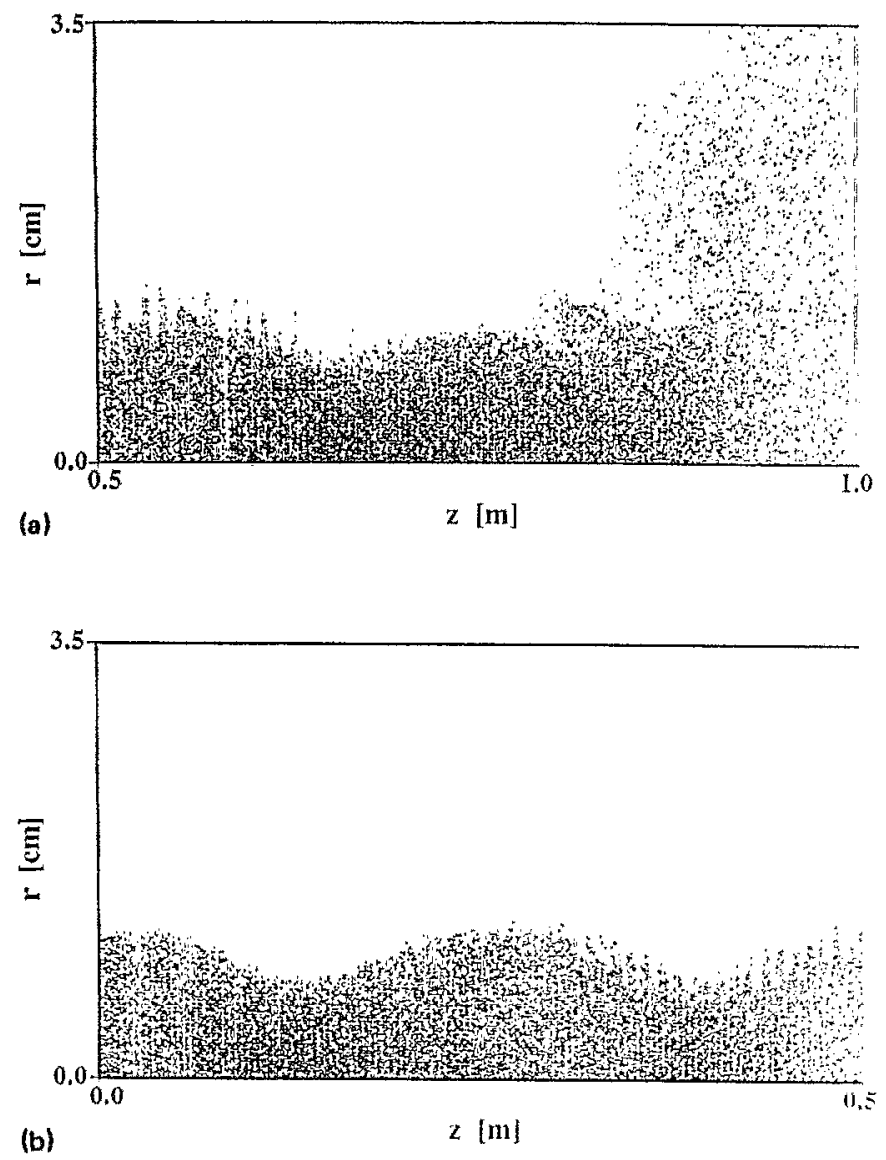

FIG. 9. Beam electron positions at $t=3.3 \mathrm{nsec}$ for an initial plasma prefill corresponding to $f_{t}=1.0$ and mismatched injection radius $\left(R_{\mathrm{tm})} \neq R_{(\mathrm{f}}\right)$. (a) $0.5<z<1.0(\mathrm{~m})$. (b) $0<z<0.5(\mathrm{~m})$.

in the beam's transverse pressure and the restoring force provided by the ion channel.

The beam's transverse momenta in the region $0<z<0.5$ $\mathrm{m}$ at $t=3.3 \mathrm{nsec}$ are given in Fig. 10. This figure shows that the oscillations in the beam envelope are accompanied by oscillations in the transverse temperature. An examination of Figs. 9 and 10 reveals that oscillations in the beam radius and the transverse temperature are out of phase so that where the beam radius is the smallest the transverse temperature is the largest, and vice versa. This behavior can be understood from Eq. (32) since beam emittance is conserved for a system of noninteracting particles in a harmonic potential well. ${ }^{32}$

The next set of simulation results are those of an underionized channel with $f_{i}=0.1$, representing a point to the left of the first peak in Fig. 1. For comparison with previous results the beam parameters are held fixed so that the only difference between these simulations and the previous ones are that $f_{i}$ has been decreased from 1.0 to 0.1 . The positions of the beam and plasma electrons at $t=3.3 \mathrm{nsec}$ are shown in Figs. 11 and 12, respectively. The positions of the beam electrons show that the envelope opens very quickly after injection, resulting in a large loss of beam current at the drift tube wall. Since $f_{i} \gg 1 / \gamma_{0}^{2}$ there are more than enough ions to 


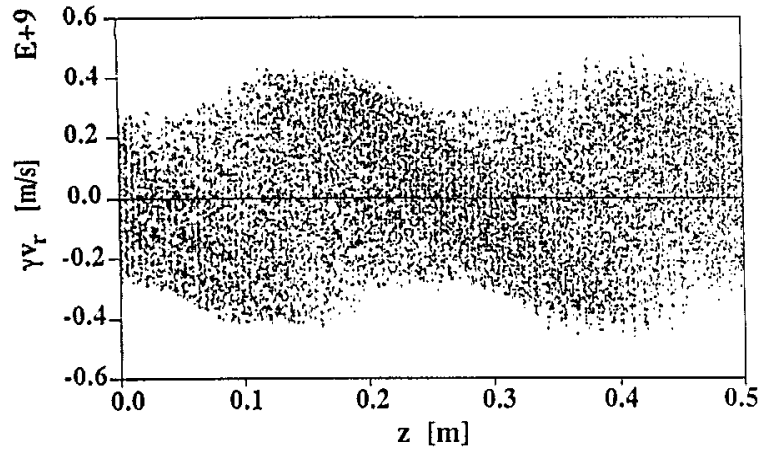

FIG. 10. Radial momentum $\left(\gamma v_{r}\right)$ in the pinched beam region $0<z<0.5$ $(\mathrm{m})$ at $t=3.3 \mathrm{nsec}$ for the beam electrons for an initial plasma prefill corresponding to $f_{1}=1.0$ and mismatched injection radius $\left(R_{\text {inj }} \neq R_{0}\right)$.

neutralize the repulsive force on the beam from self-fields. However, as Eq. (27) shows, the net ion charge is insufficient to counteract the beam's transverse pressure. Some electrons reach the end of the drift tube, but the efficiency is very much reduced compared with the previous two examples.

The axial momenta of the beam electrons in the underionized channel at $t=3.3 \mathrm{nsec}$ are shown in Fig. 13. In contrast to the axial momentum in the $f_{i}=1.0$ channel, the axial
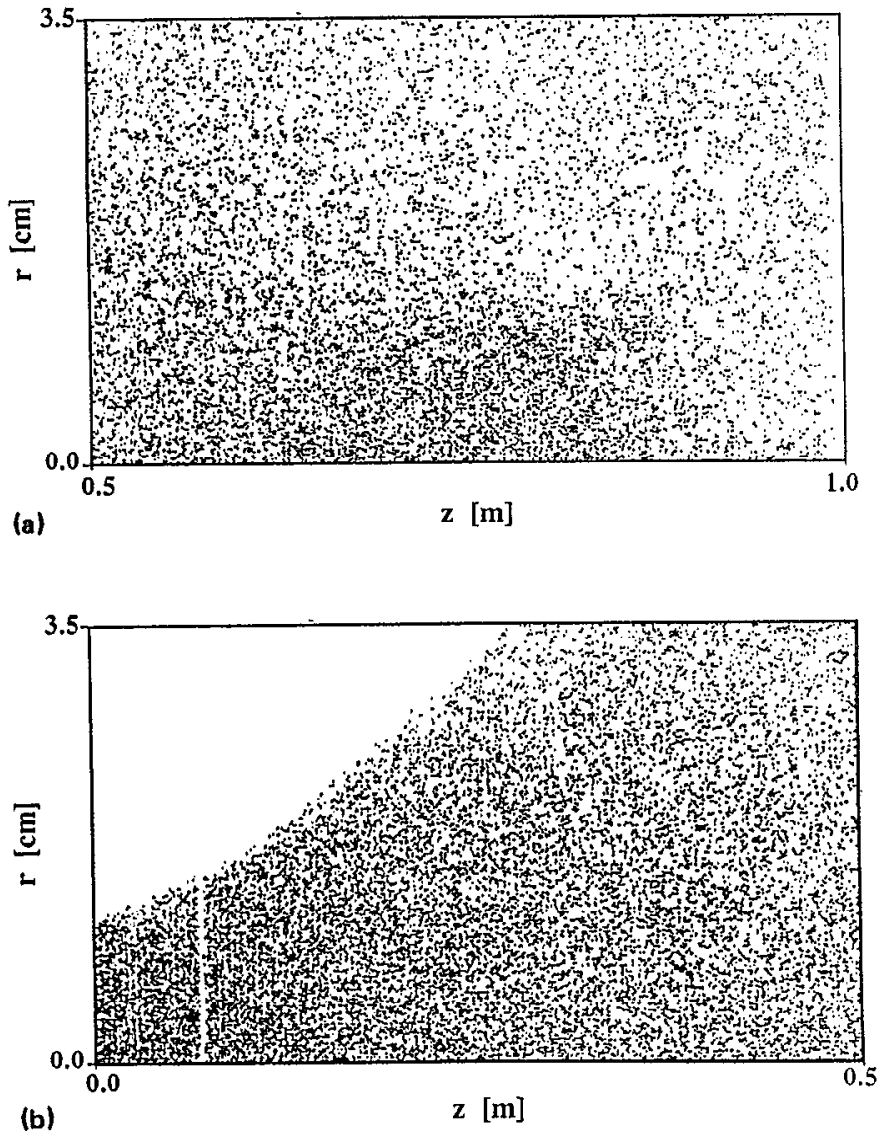

FIG. 11. Beam electron positions at $t=3.3 \mathrm{nsec}$ for an initial plasma prefill corresponding to $f_{i}=0.1$. (a) $0.5<z<1.0$ (m). (b) $0<z<0.5$ (m).

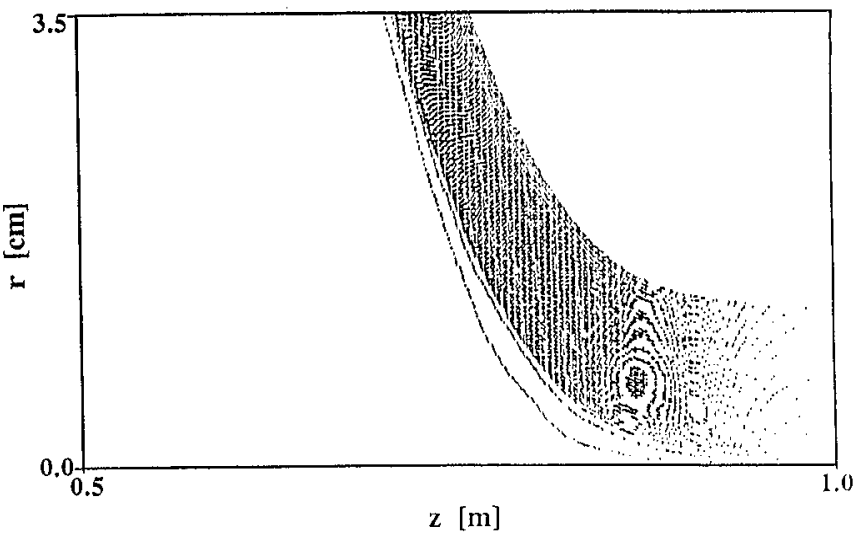

FIG. 12. Plasma electron positions in the region $0.5<z<1.0(\mathrm{~m})$ at $t=3.3$ nsec for an initial plasma prefill corresponding to $f_{t}=0.1$.

momentum of the underionized channel shows very little axial energy loss. Since the total mass of the plasma channel is ten times smaller in the $f_{i}=0.1$ case, the net kinetic energy of the plasma electrons is much smaller as they leave the channel. Hence the energy needed to create the channel is smaller and beam energy losses are correspondingly smaller.

When $f_{i}$ is larger than unity, it is possible for a large number of plasma electrons to be confined in the channel. In this case, the plasma electrons and beam electrons can inter-
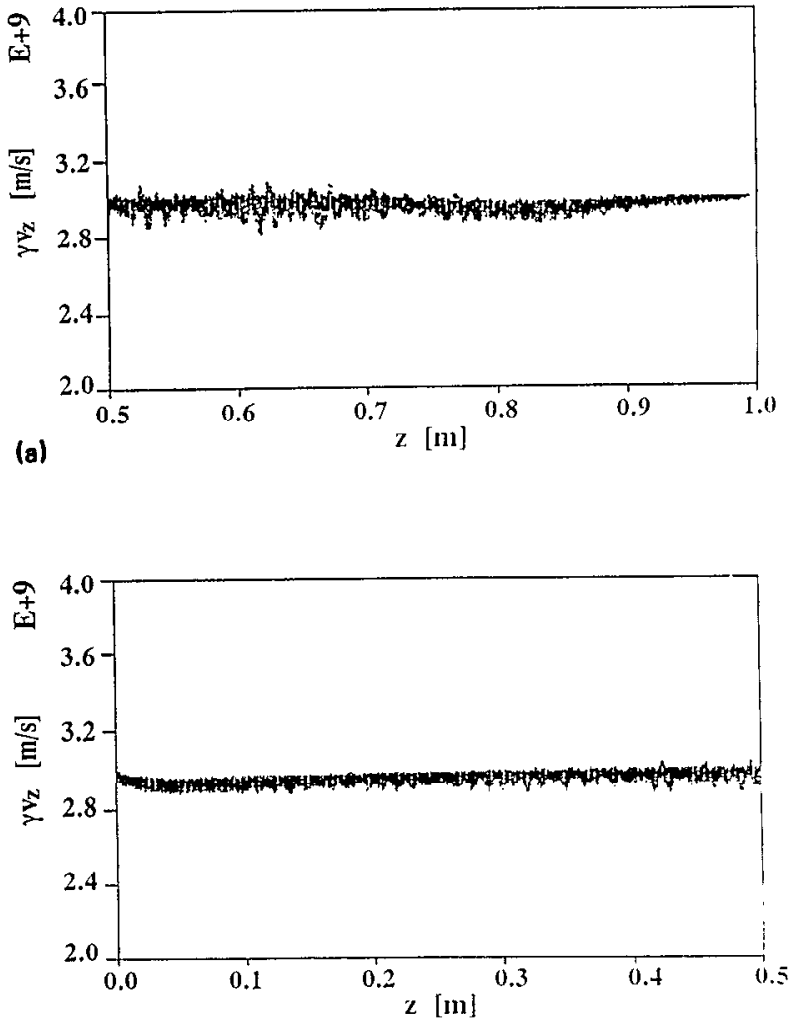

(b)

FIG. 13. Axial momentum $\left(\gamma v_{z}\right)$ at $t=3.3 \mathrm{nsec}$ for the beam electrons for an initial plasma prefill corresponding to $f_{1}=0.1$. (a) $0.5<z<1.0(\mathrm{~m})$. (b) $0<z<0.5(\mathrm{~m})$. 
act, resonantly leading to transverse oscillations that can disrupt efficient beam transport. Since the time scale for the development of the electron-electron interaction is too short for ions to participate, ions are treated in the analysis as a fixed charge-neutralizing background. For simplicity it is assumed that the beam and plasma completely fill the drift tube and, to be consistent with the symmetry assumed in the MAGIC simulations, azimuthal perturbations are ignored. From the cold fluid equations the linear electrostatic dispersion relation for the eigenfrequencies, $\omega$, can be written as $^{33,34}$

$$
k_{1}^{2} \epsilon_{1}\left(k_{z}, \omega\right)+k_{z}^{2} \epsilon_{\|}\left(k_{z}, \omega\right)=0,
$$

where $k_{1}$ is the effective transverse wave number, $k_{z}$ is the axial wave number, and $\epsilon_{1}$ and $\epsilon_{\|}$are given by

$$
\begin{aligned}
& \epsilon_{1}\left(k_{z}, \omega\right)=1-\frac{\omega_{p e}^{2}}{\omega^{2}}-\frac{\omega_{p b}^{2} / \gamma_{0}}{\left(\omega-k_{z} c \beta_{b z}\right)^{2}}, \\
& \epsilon_{\|}\left(k_{z}, \omega\right)=1-\frac{\omega_{p e}^{2}}{\omega^{2}}-\frac{\omega_{p b}^{2} / \gamma_{0}^{3}}{\left(\omega-k_{z} c \beta_{b z}\right)^{2}} .
\end{aligned}
$$

When the beam and plasma fill the drift tube, the transverse wave number satisfies the relation $k_{1} r_{b}=\mu_{m}$, where $\mu_{m}$ is the $m$ th zero of $J_{0}(x)$, the zeroth-order Bessel function of the first kind. Note that when $k_{z} \gg k_{1}$ the dispersion relation reduces to that obtained in a one-dimensional treatment.

An approximate form of the dispersion relation near the two-stream resonance can be derived by substituting $\omega=\omega_{p e}+\delta_{0}=k_{z} c \beta_{b z}+\delta_{0}$ into Eq. (35) and expanding about $\delta_{0} \ll \omega_{p e}$. Near the two-stream resonance, Eq. (35) has an approximate solution corresponding to instability, which can be written as

$\delta_{0}=\left(\frac{\gamma_{0}^{2} k_{1}^{2}+k_{z}^{2}}{k_{1}^{2}+k_{z}^{2}}\right)^{1 / 3}\left[\frac{\omega_{p e}}{\gamma_{0}}\left(\frac{\hat{n}_{b 0}}{2 n_{e 0}}\right)^{1 / 3}\left(-\frac{1}{2}+i \frac{\sqrt{3}}{2}\right)\right]$.

The first term in parentheses arises from the effects of finite radial geometry, whereas the term in brackets is identical to the result attained in a one-dimensional treatment. One consequence of finite radial geometry is the weak dependence of the peak growth rate on $\gamma_{0}$. For large beam energies $\mathrm{Eq}$. (38) shows that the peak growth rate goes to zero as $\gamma_{0}^{-1 / 3}$ not as $\gamma_{0}^{-1}$, as predicted by a one-dimensional analysis.
As a specific numerical example consider the transport of a beam with parameters given at the beginning of this section in a plasma channel with $f_{i}=6.0$. During the transient period it is assumed that the beam displaces enough plasma electrons to achieve charge neutrality. Therefore when $f_{i}=6.0$ it is assumed that the plasma electron density is roughly five times the beam electron density, which, for this example, is given by $\hat{n}_{\mathrm{e} 0}=3.3 \times 10^{11} \mathrm{~cm}^{-3}$. The most unstable wave number in this case is $k_{z}^{*} \approx \omega_{p e} / c \beta_{b z} \approx 1.1$ $\mathrm{cm}^{-1}$, which corresponds to a wavelength of approximately $5.8 \mathrm{~cm}$. The real frequency and growth rate for the most unstable fundamental mode $\left(k_{\perp} r_{b} \cong 2.4048\right)$ are $\operatorname{Re}(\omega) \cong 2.9 \times 10^{10} \mathrm{sec}^{-1}$ and $\operatorname{Im}(\omega) \approx 5.6 \times 10^{9} \mathrm{sec}^{-1} \mathrm{cor}-$ responding to a linear frequency and growth time given by $v=\operatorname{Re}(\omega / 2 \pi) \approx 4.67 \mathrm{GHz}$ and $\tau=1 / \operatorname{Im}(\omega) \approx 0.18 \mathrm{nsec}$. These values compare well with the exact numerical solutions of the dispersion relation shown in Fig. 14. The growth time is important since it provides the expected time scale for small-amplitude perturbations to become large enough to cause a breakdown of linear theory.

The next set of simulation results are those of an overionized channel with $f_{i}=6.0$ representing a point to the right of the first peak in Fig. 1. The beam electron positions at $t=3.3 \mathrm{nsec}$ for this case are shown in Fig. 15. The most notable feature of the trajectories is the large transverse oscillation in the beam electron orbits that occur over the length of the channel. These oscillations begin shortly after the beam electrons are injected at $z=0$ and continue to grow in amplitude eventually become large enough to cause the beam to strike the drift tube wall resulting in a loss of transport efficiency. Evidence of nonlinear particle bunching can also be seen in Fig. 15 by the regions of high beam electron concentrations followed by regions of low concentration along the $z$ direction. The large forces responsible for these transverse oscillations are interpreted as those arising from the two-stream instability. This interpretation will be substantiated in the remainder of this section by comparing the wavelength and frequency of this oscillation with that obtained by linear theory.

The axial momenta of the beam electrons at $t=3.3 \mathrm{nsec}$ in the overionized channel are shown in Fig. 16. From this figure a very well-defined oscillation wavelength of approximately $5.5 \pm 0.2 \mathrm{~cm}$ is evident. This is in good agrecment

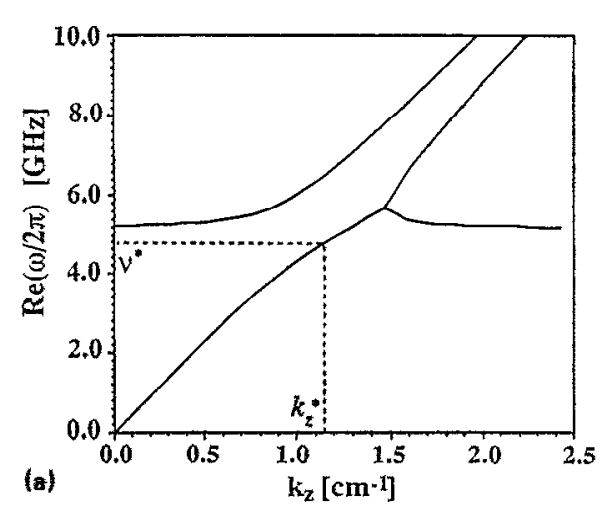

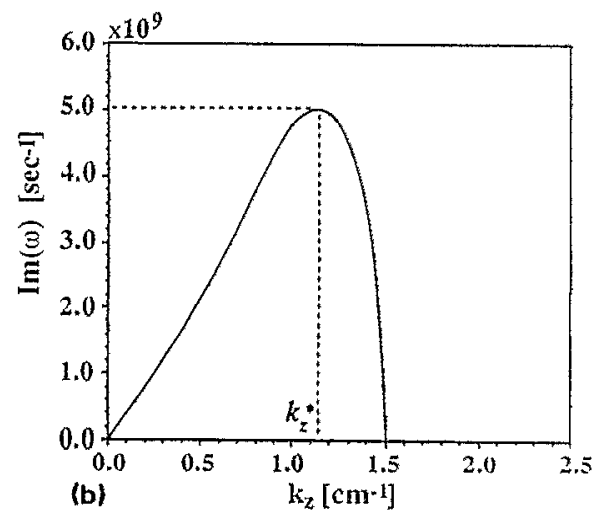

FIG. 14. Numerical solution to the electrostatic dispersion equation for a plasma prefill corresponding to $f_{i}=6.0$. (a) Real frequency, (b) growth rate. 

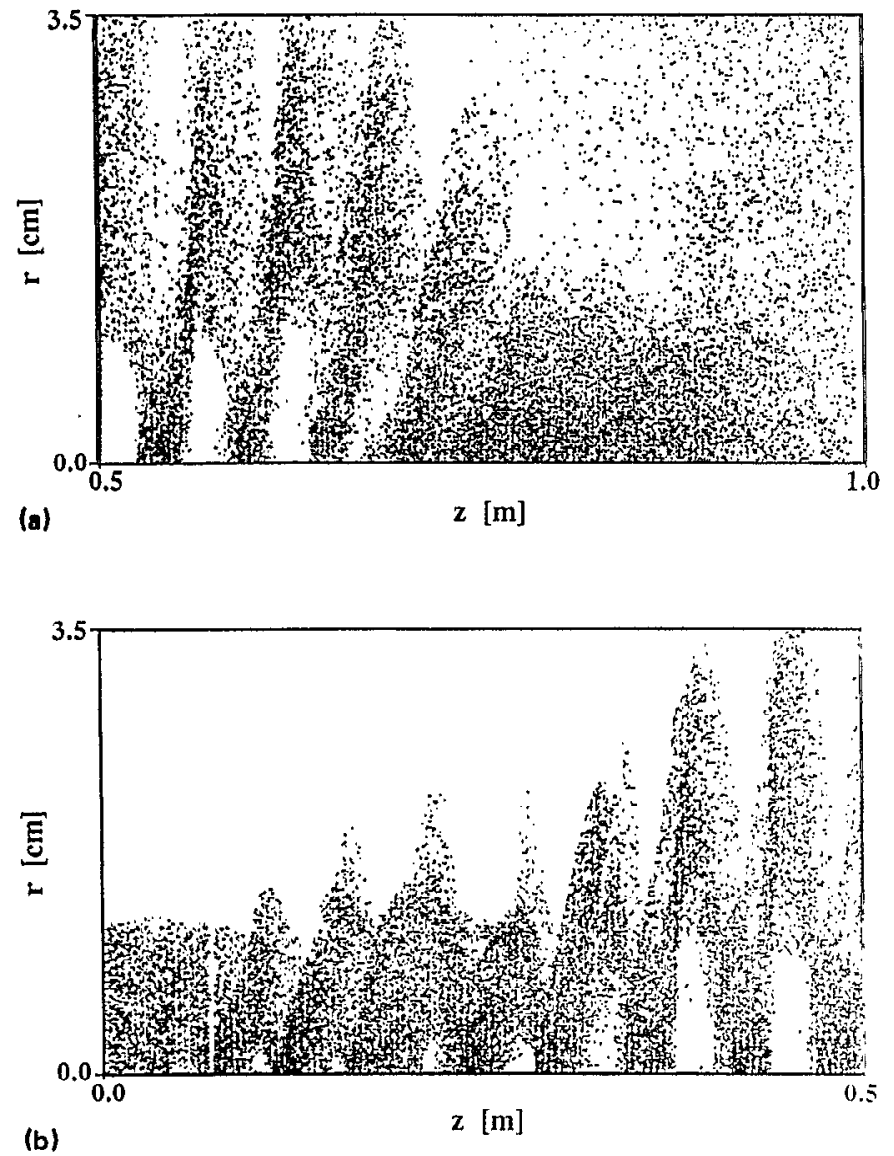

FIG. 15. Beam electron positions at $t=3.3 \mathrm{nsec}$ for an initial plasma prefill corresponding to $f_{1}=6.0$. (a) $0.5<z<1.0(\mathrm{~m})$. (b) $0<z<0.5(\mathrm{~m})$.

with the wavelength of $5.6 \mathrm{~cm}$ for the fastest growing wave predicted from linear theory. A spatial growth length of 13.7 $\mathrm{cm}$ can be estimated from this figure from which a temporal growth rate of $2.2 \times 10^{9} \mathrm{sec}^{-1}$ can be inferred. This is compared with the growth rate of $5.0 \times 10^{9} \mathrm{sec}^{-1}$ computed from linear theory. One source of the discrepancy is the many simplifications used in arriving at the dispersion relation, for example, that the beam and plasma completely fill the drift tube, purely electrostatic waves, and that pressure perturbations could be neglected. As seen from the radial momenta of the beam electrons at $t=3.3 \mathrm{nsec}$ shown in Fig. 17, the transverse pressure perturbations are not small and may be important in properly predicting the growth rate of beam transport in overionized plasma channels. Nonlinear processes such as particle trapping and wave-wave interactions act to slow down wave growth and probably also account for some of the discrepancy between observed growth rate and linear theory.

Figure 18(a) shows the radial electric field $25 \mathrm{~cm}$ downstream from the injection point at $r_{b} / 2=0.65 \mathrm{~cm}$ from the axis as a function of time. The corresponding FFT of the radial electric field is shown in Fig. 18(b). The FFT shows three distinct Fourier components: one at roughly $500 \mathrm{MHz}$, the second and dominant frequency component at about 4.5 $\mathrm{GHz}$, and a third frequency component at about $9.0 \mathrm{GHz}$. It

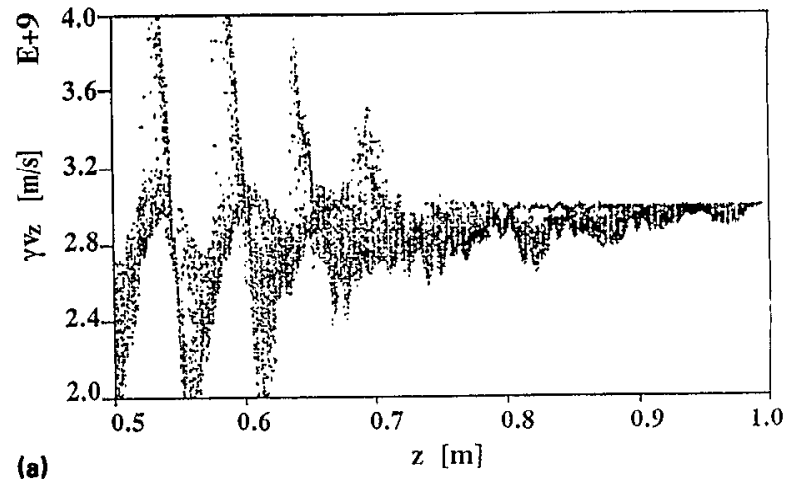

(a)

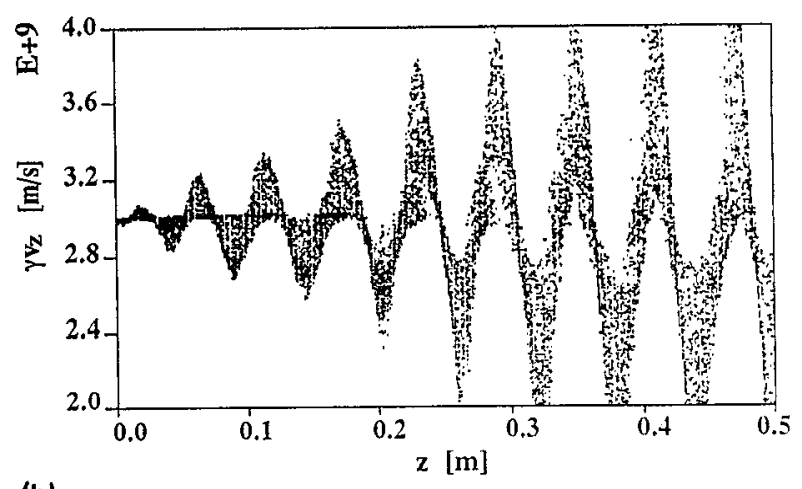

(b)

FIG. 16. Axial momentum $\left(\gamma v_{z}\right)$ at $t=3.3 \mathrm{nsec}$ for the beam electrons for an initial plasma prefill corresponding to $f_{i}=6.0$. (a) $0.5<z<1.0(\mathrm{~m})$. (b) $0<z<0.5(\mathrm{~m})$.

will be shown in the next section that $500 \mathrm{MHz}$ is very close to the frequency expected in the channel for a betatron oscillation in the beam envelope. The dominant frequency component is the $4.5 \mathrm{GHz}$ signal, which should be compared with $4.8 \mathrm{GHz}$, the frequency of the most unstable linear wave. The $9.0 \mathrm{GHz}$ signal is twice the frequency of the dominant wave and is interpreted as the second harmonic of the fundamental wave. Since harmonic generation is a nonlinear phenomenon, the existence of this wave is not with the scope of linear theory.

\section{ENVELOPE AND ORBIT ANALYSIS OF IFR TRANSPORT}

In the previous section PIC simulations of IFR transport were presented. Some of the aspects of the simulations such as the radial expansion of the beam front and the envelope oscillations are not explained by the Vlasov treatment presented in Sec. II. In this section, the beam envelope equation is used to facilitate an understanding of the PIC results and provide further insight into IFR transport phenomena. To provide a convenient way to visualize solutions to the equilibrium Vlasov equation, a test particle approach is devcloped at the end of this section. This illustrates the connection between the statistical description offered by the Vlasov 

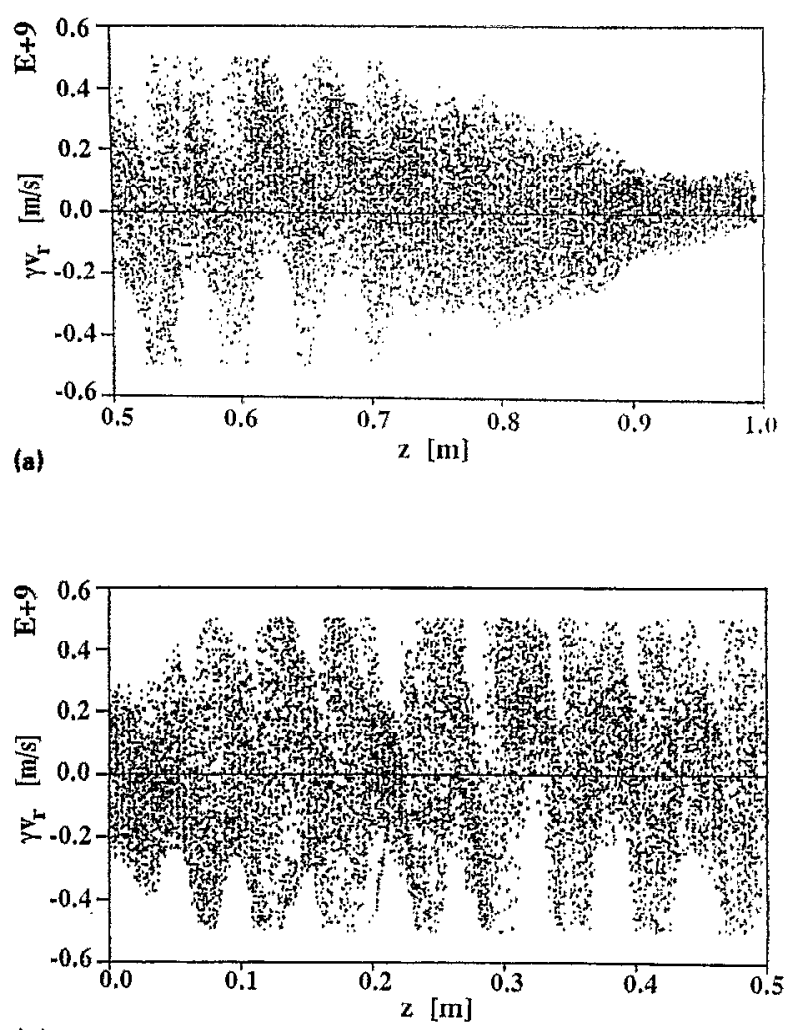

(b)

FIG. 17. Radial momentum $(\gamma$, ) at $t=3.3$ nsec for the beam electrons for an initial plasma prefill corresponding to $f=6.0$. (a) $0.5<z<1.0(\mathrm{~m})$. (b) $0<z<0.5(\mathrm{~m})$.

equation, the PIC method that follows the dynamics of a collection of individual ions and electrons in self-consistent electromagnetic fields, and the envelope approach that follows the rms radius of the beam. The end result is a very detailed picture of the dynamic force balance required for efficient IFR transport.

The beam envelope equation describes the time evolution of the rms radius of a thin axial slice of beam particles as a result of the average transverse forces acting on the beam slice. The starting point for the envelope analysis is the beam envelope equation of Lee and Cooper, ${ }^{23}$

$$
\begin{aligned}
\ddot{R}+ & \frac{\dot{\gamma}}{\gamma} \dot{R}+\frac{c^{2} U}{R}+\frac{1}{4} \omega_{c}^{2} R-\frac{c^{2}}{R^{3}} \\
\quad & \times\left(\epsilon^{2}+\int_{0}^{c \beta_{b z^{t}}} d z^{\prime} R^{2} \frac{\delta\left\langle\theta_{s}^{2}\right\rangle}{\delta z^{\prime}}\right)=0,
\end{aligned}
$$

where $R$ is the rms beam radius. Each term in the beam envelope equation has physical significance. The $\ddot{R}$ term represents the inertia of a "fictitious" particle whose trajectory traces out the rms radius of the beam slice while the forces that affect the rms radius are represented by the remaining terms in Eq. (39). The second term in Eq. (39) is analogous to friction in a mechanical system and arises from the loss of beam energy. These energy losses are often small in IFR experiments and usually confined to the initial transient pe-
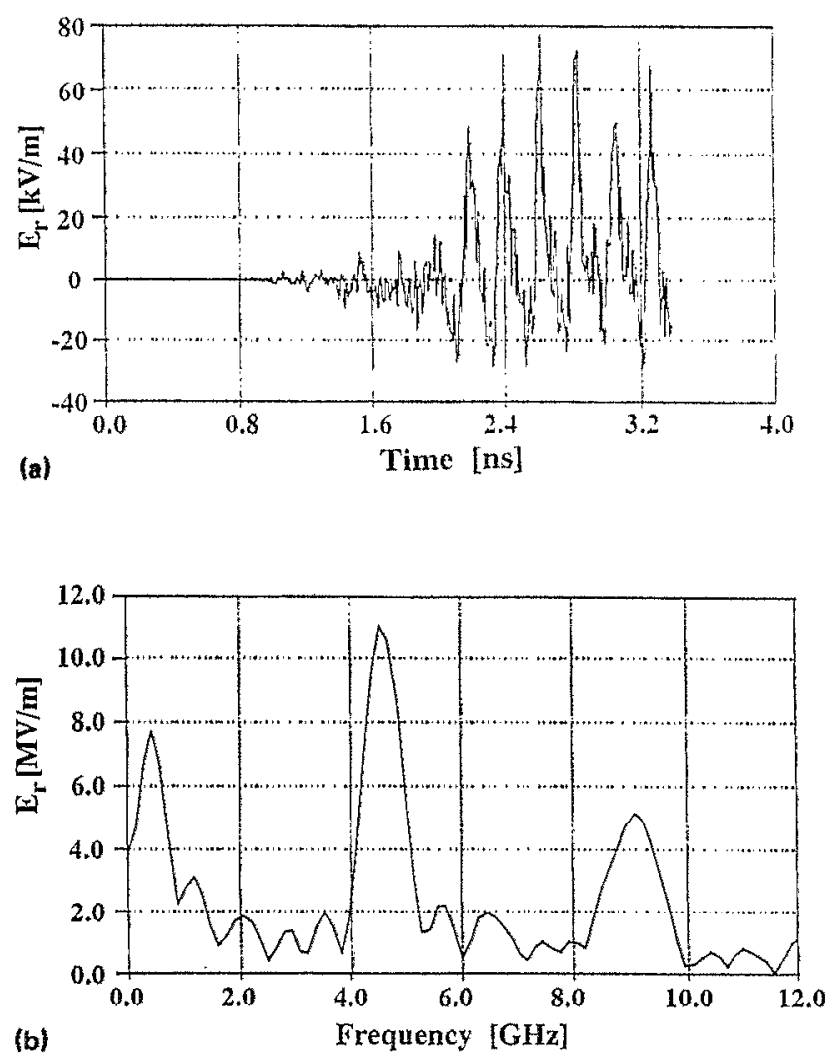

FIG. 18. (a) The radial electric field as a function of time measured at $r=0.65 \mathrm{~cm}$ and $z=25 \mathrm{~cm}$ for an initial plasma prefill corresponding to $f_{i}=6.0$. (b) The corresponding FFT of the radial electric field,

riod of the beam pulse. The third term in Eq. (39) arises from the self-electric and magnetic forces on the beam and is determined by current averaging the transverse electric and magnetic forces over the cross section of the beam. For a beam with a current density profile $J_{b 0}(r)$ this term can be expressed as

$$
\begin{aligned}
\frac{c^{2} U}{R}= & \frac{1}{R} \int_{0}^{\infty} d r \frac{2 \pi r J_{b 0}(r)}{I_{b}} \\
& \times r^{2}\left(\frac{e}{\gamma_{0} m_{e}} \frac{\left[E_{r 0}(r)-\beta_{b z} B_{\theta 0}(r)\right]}{r}\right) .
\end{aligned}
$$

It should be noted that the term in parentheses has the units of frequency and, for a uniform beam in a channel of uniformly distributed immobile ions, the self-electric and magnetic fields are linear in $r$. In this case the term in parentheses is independent of $r$ and is given by $\Omega_{\beta}^{2}$ [see Eq. (22)] so that Eq. (40) reduces to

$$
c^{2} U / R=R \Omega_{\beta}^{2}
$$

The fourth term in Eq. (39) arises from the focusing force provided by an external magnetic field. Since IFR experiments use little or no external guide fields this term is neglected in the analysis. The last term in Eq. (39) accounts for increases in the rms beam radius arising from the transverse beam pressure. In this term, the injected beam emittance is 
represented by $\epsilon^{2}$ while the integration accounts for emittance growth due to beam scatterings in the plasma and background gas.

The mean-squared scattering angle per unit length in the background gas can be estimated by assuming that multiple small angle Rutherford scattering is the dominant mechanism for emittance growth. In this case, the mean-squared scattering angle per unit length is independent of $z$ and can be expressed as ${ }^{35}$

$$
\begin{aligned}
\frac{\delta\left\langle\theta_{s}^{2}\right\rangle}{\delta z}= & \frac{8 \pi r_{e}^{2} n_{g} Z^{2}}{\gamma_{0}^{2} \beta_{b z}^{4}} \ln \left(\frac{\theta_{\min }}{\theta_{\max }}\right) \\
& \cong 2.0 \times 10^{-24} \frac{n_{g} Z^{2}}{\gamma_{0}^{2} \beta_{b z}^{4}} \ln \left(204 Z^{-1 / 3}\right),
\end{aligned}
$$

where $Z$ is the atomic number of the gas, $n_{g}$ is the neutral gas density in $\mathrm{cm}^{-3}, r_{e}=e^{2} / m_{e} c^{2}$ is the classical electron radius, and $\theta_{\min }$ and $\theta_{\max }$ are the minimum and maximum scattering angles. Using Eq. (42) as an estimate for the meansquared scattering angle, a first-order approximation for emittance growth due to multiple small angle scatterings in the background gas can be calculated by assuming $R \cong R_{\mathrm{inj}}$ and performing the integration in Eq. (40) to yield

$$
\begin{aligned}
\int_{0}^{L_{z}} d z^{\prime} R^{2} \frac{\delta\left\langle\theta_{s}^{2}\right\rangle}{\delta z^{\prime}} \cong & 2.0 \times 10^{-24} \frac{n_{g} Z^{2}}{\gamma_{0}^{2} \beta_{b z}^{4}} \\
& \times \ln \left(204 Z^{-1 / 3}\right) R_{\mathrm{inj}}^{2} L_{z},
\end{aligned}
$$

where $L_{z}=c \beta_{b z} \tau$ is the distance traveled by the beam in the gas in time $\tau$. This last equation is valid when changes in emittance from gas scattering are small compared to the initial beam emittance and the injected rms beam radius is close to the rms beam radius averaged over $L_{z}$. A scale length for emittance growth can be defined by requiring that Eq. (43) be $10 \%$ of the initial beam emittance. With this definition and using Eq. (34) to eliminate $R_{\text {inj }}$ one can arrive at the following scale length for emittance growth from multiple small angle scatterings in the background gas,

$$
L_{z} \cong 3.0 \frac{\gamma_{n} \beta_{b z}^{4} T_{10}}{p Z^{2} \ln \left(204 Z^{-1 / 3}\right)},
$$

where $p$ is the gas pressure in Torr (at room temperature $n_{g} \cong 3.2 \times 10^{16} \mathrm{p}$ ), $L_{z}$ is in $\mathrm{cm}$, and $T_{10}$ is in eV. For a $5 \mathrm{MeV}$ beam with a transverse temperature of $50 \mathrm{keV}$ in 0.1 Torr of argon $(Z=18)$, the scale length for emittance growth predicted by Eq. (44) is $116 \mathrm{~m}$. In 10 Torr of argon the scale length decreases to $1.16 \mathrm{~m}$. Since this paper is primarily concerned with IFR transport over several meters in a low pressure gas ( $p<0.1$ Torr), beam scattering in the background gas is neglected in the subsequent analysis. However, as this example illustrates, emittance growth from multiple small angle scatterings can be important in the high-pressure transport regime.

With the simplifying assumptions outlined in the preceding paragraphs, the beam envelope equation relevant to IFR beam transport can be simplified to

$$
\beta_{b z}^{2} \frac{d^{2} R}{d z^{2}}+\frac{\Omega_{\beta}^{2}}{c^{2}} R-\frac{\epsilon^{2}}{R^{3}}=0,
$$

where $z=c \beta_{b z} t$ has been substituted as the independent variable. The equilibrium rms radius predicted by Eq. (45) is given by

$$
R_{0}^{4}=\frac{c^{2}}{\Omega_{\beta}^{2}} \epsilon^{2} \cong \frac{c^{2}}{\Omega_{\beta}^{2}} R_{0}^{2}\left(\frac{T_{\perp 0}}{\gamma_{0} m_{e} c^{2}}\right),
$$

where the second form assumes that the rms injected radius is well matched with the equilibrium radius. For a uniform channel the rms radius is related to the beam radius by $R_{0}=r_{b} / \sqrt{2}$ so that Eqs. (24) and (46) produce the same estimate for beam radius.

The usual procedure in the envelope analysis is to linearize Eq. (45) about $R_{0}$ and solve the resulting linear equation for small oscillations about the equilibrium rms radius. In contrast to this approach, a procedure for constructing exact solutions to Eq. (45) will be outlined. This not only produces a more general result but provides more physical insight into the nature of the envelope oscillations. If the betatron frequency is independent of $z$ then

$$
H=\frac{1}{2}\left(\frac{d R}{d z}\right)^{2}+\frac{1}{2} k_{\beta}^{2} R^{2}+\frac{1}{2} \frac{\epsilon^{2}}{R^{2} \beta_{b z}^{2}}
$$

is a constant of the motion, where $k_{\beta}=\Omega_{\beta} / c \beta_{b z}$ is the betatron wave number. Equation (47) is a statement of energy conservation for the pseudoparticle. The first term in Eq. (47) is related to the kinetic energy of the pseudoparticle, the second term represents the potential energy of the beam in the equilibrium electric and magnetic fields in the channel, and the third term is related to the transverse thermal energy of the beam. Insight into the nature of the equilibrium can be gained by noting that, when $d R / d z=0, R=R_{0}$ and the two remaining terms in Eq. (47) are equal. Therefore in IFR equilibria the net transverse energy is partitioned equally between the beam's potential energy in the channel electric and magnetic fields and the transverse thermal energy of the beam.

An exact equation for the beam envelope can bc obtained from Eq. (47) by separating variables and integrating to give

$$
z=\int_{R_{\text {inj }}}^{R(z)} \frac{R d R}{\left(2 H R^{2}-k_{\beta}^{2} R^{4}-\epsilon^{2} / \beta_{b z}^{2}\right)^{1 / 2}} .
$$

This equation can be integrated to yield an implicit equation for $R(z)$, which can then be inverted and expressed as

$$
R(z)=\frac{1}{k_{\beta}}\left(H+\left(H-k_{\beta}^{2} R_{\mathrm{inj}}^{2}\right) \frac{\sin \left(2 k_{\beta} z-\theta_{0}\right)}{\sin \left(\theta_{0}\right)}\right)^{1 / 2},
$$

where the initial phase angle $\theta_{0}$ is given by

$$
\sin \left(\theta_{0}\right)=\frac{H-k_{\beta}^{2} R_{\mathrm{inj}}^{2}}{\left(H-k_{\beta}^{2} \epsilon^{2} / \beta_{b z}^{2}\right)^{1 / 2}} .
$$

One of the interesting features of Eq. (49) is that the nonlinear envelope oscillations all have the same wavelength $\lambda=\lambda_{\beta} / 2$, where $\lambda_{\beta}=2 \pi / k_{\beta}$ is the betatron wavelength. The constant $H$ can be evaluated from Eq. (47) in terms of the injected $\mathrm{rms}$ beam radius and slope at $z=0$. For the special case where $d R / d z=0$ at $z=0, H$ can be expressed as 


$$
H=\frac{1}{2} k_{\beta}^{2} R_{\mathrm{inj}}^{2}\left(1+R_{0}^{4} / R_{\mathrm{inj}}^{4}\right),
$$

and Eq. (47) simplifies to

$R(z)=\frac{R_{\mathrm{inj}}}{\sqrt{2}}\left[\left(1+\frac{R_{0}^{4}}{R_{\mathrm{inj}}^{4}}\right)+\left(1-\frac{R_{0}^{4}}{R_{\mathrm{inj}}^{4}}\right) \cos \left(2 k_{\beta} z\right)\right]^{1 / 2}$.

Note that if the injection radius is well matched to the equilibrium radius then $\mathrm{Eq}$. (52) shows that $R(z)=R_{0}$ and is independent of $z$. Furthermore, for small perturbations from equilibrium it is possible to show that the results obtained from the linearization of Eq. (45) are recovered.

Figure (19) shows a plot of the beam envelope computed from Eq. (52) for a beam-plasma channel with the parameters $\hat{n}_{b 0}=6.65 \times 10^{10} \mathrm{~cm}^{-3},\left(\gamma_{0}-1\right) m_{e} c^{2}=5 \mathrm{MeV}$, and $f_{i}=1$ with injection radii of $0.5,1.1$, and $2.0 \mathrm{~cm}$. For this choice of parameters the oscillation frequency and wavelength are $v_{\beta}=\Omega_{\beta} / 2 \pi \cong 500 \mathrm{MHz}$ and $\lambda=\lambda_{\beta} / 2 \cong 30 \mathrm{~cm}$, which agrce well with the oscillation frequency and wavelength observed in the simulations. Recall that the equilibrium radius for this example was $r_{b}=\sqrt{2} R_{0}=1.3 \mathrm{~cm}$. Notice that when the injection radius closely matches the equilibrium radius, as in the $1.1 \mathrm{~cm}$ case, the oscillations are small and a tight beam pinch is maintained. However, if the injection radius does not closely match the equilibrium radius, as in the 0.5 and $2.0 \mathrm{~cm}$ cases, the oscillations are not small and the tight beam pinch is lost. The large amplitude of these oscillations can be traced to imbalances in the transverse forces on the beam. To get a better understanding of this force imbalance, consider the $0.5 \mathrm{~cm}$ injection case shown in Fig. 19. As the beam enters the channel the forces due to beam pressure greatly exceed the restoring force of the ion channel causing the beam envelope to rapidly expand. As the beam expands the transverse kinetic pressure decreases and the restoring force provided by the ion channel increases. Since the beam envelope has inertia as it moves through the point of zero force it continues to expand. However, past the equilibrium point the restoring force from the ion channel exceeds the transverse pressure and envelope expansion slows down and eventually comes to rest. Since the focusing force now greatly exceeds the transverse pressure, the beam envelope pinches past the equilibrium point and the whole process repeats itself.

The PIC simulations show that the transverse focusing

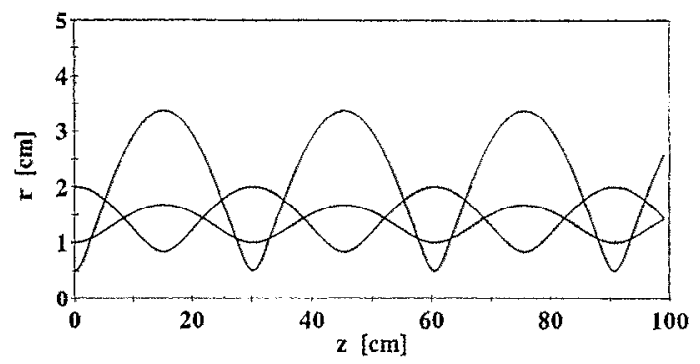

FIG. 19. Solutions to the beam envelope equation for three different injection radii $\left(r_{\mathrm{m},}=0.5,1.1\right.$, and $\left.2.0 \mathrm{~cm}\right)$ for a beam-plasma channel whose equilibrium radius is $1.3 \mathrm{~cm}$. force is small during the time that it takes for plasma electrons to be accelerated from the channel. This implies that the betatron frequency is small at the beam front and rises to its full value in the main body of the beam. A heuristic model of the beam front physics can be obtained by letting the focusing force vary from slice to slice in the beam envelope equation. In this situation the equation of motion for the rms radius each beam slice is given by replacing $\Omega_{\beta}^{2}$ in Eq. (45) with $\alpha_{j} \Omega_{\beta}^{2}$, where $\alpha_{j}$ is much smaller than one at the beam head and unity in the main body of the beam. The rms radius for the $j$ th beam slice then becomes

$$
\begin{aligned}
R_{j}(z)= & \frac{R_{\mathrm{inj}}}{\sqrt{2}}\left[\left(1+\frac{\epsilon^{2}}{\alpha_{j} k_{\beta}^{2} R_{\mathrm{inj}}^{4}}\right)\right. \\
& \left.+\left(1-\frac{\epsilon^{2}}{\alpha_{j} k_{\beta}^{2} R_{\mathrm{inj}}^{4}}\right) \cos \left(2 \sqrt{\alpha_{j}} k_{\beta} z\right)\right]^{1 / 2} .
\end{aligned}
$$

In the limit that $2 \sqrt{\alpha_{j}} k_{\beta} z \ll 1$, Eq. (53) reduces to

$$
R_{j}(z) \approx R_{\text {inj }}\left(1+\epsilon^{2} z^{2} / R_{\text {inj }}^{4}\right)^{1 / 2} .
$$

This shows that the beam frecly expands due to its finite emittance when the pinch force is weak. To obtain a snapshot of the rms beam radius over the entire length of the channel one must string together envelope solutions for many beam slices. Results from this calculation will be shown in conjunction with the test particle analysis for $\alpha_{j}=F_{\beta}\left(\xi_{j}\right)$, where $\xi_{j}$ locates the distance of the $j$ th slice behind the beam head. The shape for $F_{\beta}$ used in these calculations is shown in Fig. 20.

Since electron bcams are ultimately comprised of many individual electrons, it is important to understand IFR transport on the basis of an individual beam electron. In this section it is seen that a small number of electron trajectories not only provide a useful way to visualize solutions of the Vlasov-Maxwell equations but also provide a useful connection between solutions of the Vlasov-Maxwell equations, the beam envelope equation, and the PIC model. Under the assumption of the paraxial approximation and in the small $v / \gamma$ limit, the equations of motion for an individual test particle in a uniform beam-plasma channel simplify to

$$
\beta_{b z}^{2} \frac{d^{2} r_{i}}{d z^{2}}+\frac{\alpha_{i} \Omega_{\beta}^{2}}{c^{2}} r_{i}-\frac{L_{i}^{2}}{r_{i}^{3}}=0,
$$

where $r_{i}$ and $L_{i}=r_{i} \beta_{\theta}$ are the radial position and constant

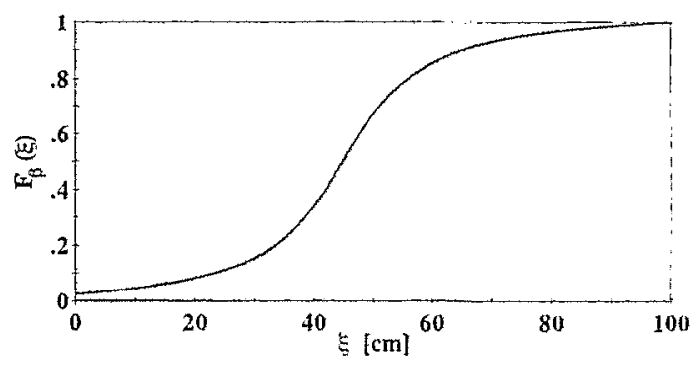

FIG. 20. Axial shape function used in the beam envelope equation and test particle analysis to heuristically model the beam front physics at $t=3.3$ nsec. 
angular momentum of the $i$ th test particle and $\alpha_{i}$ controls the strength of the focusing force. A comparison of the beam envelope equation [Eq. (45)] and the test particle equation of motion [Eq. (55)] reveals that they are very similar in form. In the solution of Eq. (55), however, the initial radial position and transverse velocity of each test particle are chosen by sampling the distribution function. Since, in general, each test particle has a different angular momentum, $L_{i}$ varies from one test particle to the next. In this paper the initial conditions of the test particles are determined by sampling the distribution given in Eq. (17). As shown in previous sections, this distribution represents a beam with uniform density and parabolic temperature profiles. In contrast the beam envelope equation [Eq. (45)] determines the average behavior of all the particles in a beam slice, whereas Eq. (55) follows the trajectory of a particular particle in that beam slice.

Since Eqs. (45) and (55) are similar in form, the solution to Eq. (55) is constructed in a similar manner and can be written as

$$
\begin{aligned}
r_{i}(z)= & \frac{1}{\sqrt{\alpha_{i}} k_{\beta}}\left[H_{i}+\sqrt{H_{i}^{2}-\alpha_{i} k_{\beta}^{2} L_{i}^{2}}\right. \\
& \left.\times \sin \left( \pm 2 \sqrt{\alpha_{i}} k_{\beta} z-\theta_{0}\right)\right]^{1 / 2}
\end{aligned}
$$

where

$$
H_{i}=\frac{1}{2} \beta_{10}^{2}\left(r_{0}\right)+\frac{1}{2} k_{\beta}^{2} r_{0}^{2}
$$

is the normalized single particle energy and

$$
\theta_{0}=\sin ^{-1}\left(\frac{H_{i}-\alpha_{i} k_{\beta}^{2} r_{0}^{2}}{\sqrt{H_{i}^{2}-\alpha_{i} k_{\beta}^{2} L_{i}^{2}}}\right)
$$

is the initial phase angle. In Eq. (56) the positive sign is taken if the initial radial velocity of the particle is positive and the negative sign is taken if the initial radial velocity is negative. The transverse velocity that appears in Eq. (57) is given by

$$
\beta_{\perp 0}^{2}\left(r_{0}\right)=T_{b 1}\left(r_{0}\right) / \gamma_{0} m_{e} c^{2},
$$

where the transverse temperature is given in Eq. (26).

Figure 21 shows the solution of Eq. (56) for six streams of test electrons in a transport channel that is well matched to the equilibrium radius. Each solid line in the figure represents a stream of test electrons created at $z=0$ with identical initial conditions. The dashed line in this figure is the beam envelope obtained from stringing many solutions to the en-

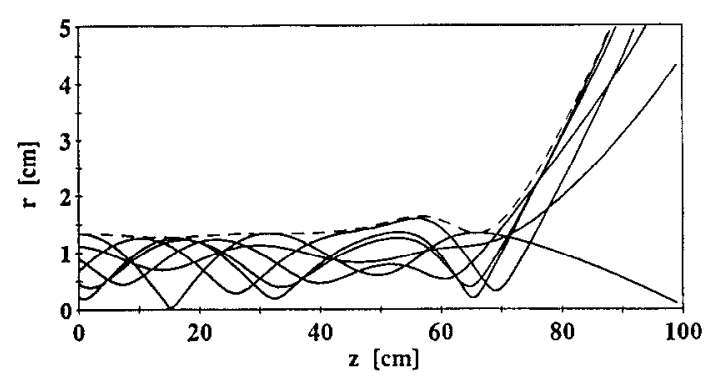

FIG. 21. Test particle trajectories in the beam-plasma channel for an injection radius that is well matched to the equilibrium radius. velope equation together for many beam slices. This figure demonstrates the relationship between the three apparently different models used in this paper to describe IFR transport; the Vlasov-Maxwell model presented in Sec. II, the PIC treatment presented in Sec. III, and the envelope treatment presented in this section. Since all the beam electrons are contained within the envelope, the interpretation of the beam envelope as the turning point in the radial orbits of the individual beam electrons is justified. Figure 21 further illustrates the expansion of the beam envelope due to force imbalances at the beam front. This behavior agrees qualitatively with the PIC results of Sec. III.

The trajectories of six streams of test electrons for which the injected radius is mismatched with the equilibrium radius are shown by the solid curves in Fig. 22. The beam envelope is again represented by the dashed line in the figure. From this figure it is seen that the trajectories of all six streams of test particles are contained within the envelope. Notice that the transverse beam pressure (as deduced by the slope of the test particle trajectories) is the largest where the beam pinch is the tightest and smallest where the beam pinch is weak. Therefore the explanation of the envelope oscillation as an exchange between the transverse thermal energy of the beam and the potential energy of the beam in the focusing channel is justified.

\section{SUMMARY AND CONCLUSION}

This paper has presented three distinct models of REB transport in low-density plasma channels: Vlasov-Maxwell theory, PIC computer simulations, and a beam envelope analysis. The equilibrium solutions to the relativistic Vlasov-Maxwell equations show that efficient IFR transport is maintained by providing a sufficient number of ions to balance radial beam expansion caused by the radial self-electric field and transverse beam pressure. Equation (30) gives the number of ions necessary to achieve this balance and it is shown that this condition is insensitive to the details of the assumed functional form for the distribution. To avoid disruptive electron-electron instabilities and also provide radial confinement the charge neutralization fraction computed from Eq. (30) should also satisfy $f_{i} \leqslant 1$.

PIC simulations of IFR transport performed in situations where $f_{i}$ satisfies Eq. (30) show that, after an initial transient period, the beam relaxes into a confined equilibri-

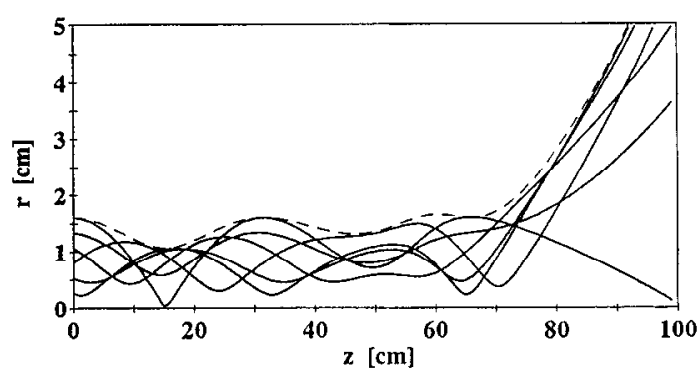

FIG. 22. T'est particle trajectories in the beam-plasma channel for an injection radius that is not well matched to the equilibrium radius. 
um that is maintained over a large portion of the channel. When the injected beam radius is mismatched from the equilibrium radius, PIC simulations show envelope oscillations that cause by transverse force imbalance. These oscillations could lead to emittance growth through phase mixing in nonuniform beam profiles. PIC simulations performed with $f_{i}$ smaller than required by Eq. (30) show that, while the ion charge in the channel is sufficient to neutralize the space charge of the beam, it is insufficient to overcome the transverse beam pressure and fail to provide radial confinement of beam electrons. In this case, transport efficiency drops as electrons expand radially and strike the drift tube wall. PIC simulations performed with $f_{i}=6$ show growing oscillacions in the beam electron orbits; the wavelength and frequency of these oscillations agree well with a linear description of the two-stream instability. Therefore the results of the PIC simulations indicate that to achieve efficient IFR transport the charge neutralization must be large enough to provide sufficient beam confinement yet small enough to avoid the two-stream instability. The simulations presented in this paper are explicit and therefore limited by the Courant stability criterion $(\Delta t<\Delta x / c)$. This effectively limits the time scale of the simulations to several nsec and makes the simulation of electron-ion interactions difficult since the time scale for these interactions can be more than an order of magnitude larger. An additional weakness of the simulations is that they are two dimensional and miss many features of the beam-plasma channel, such as the ion hose interaction, which require a three-dimensional treatment.

In Sec. IV it was shown that emittance growth from multiple small angle scattering in the background gas is negligible and a scale length for emiltance growth is estimated for low pressure IFR transport. A method for solving the nonlinear envelope is developed that shows the oscillations in the beam envelope observed in simulations of focused but mismatched beam-plasma channels is due to an imbalance between the repulsive force provided by beam pressure and the restoring force provided by channel ions. The connections between the three apparently different models used in this paper to describe IFR transport are demonstrated by the test particle approach presented at the end of Sec. IV. It is also shown that many aspects of the IFR equilibrium can be produced with relatively few test particle streams.

In conclusion, the results of this work provide a firm theoretical foundation and predictive capability that should be beneficial to future studies of relativistic electron beam transport in the ion-focused regime.

\section{ACKNOWLEDGMENTS}

Discussions with R. F. Lucey, J. Miller, D. B. McGarrah, S. Whetstone, T. Spencer, J. Choi, and all the guys in the University of Michigan's plasma lab are gratefully acknowledged. Comments provided by R. Hubbard and R. Fernsler are also greatly appreciated.

The first author would like to acknowledge the University of Michigan Regents and the American Nuclear Society Graduate Scholarship for their support of this work and the
Air Force Office of Scientific Research for making the MAGIC code available.

'W. H. Bennett, Phys. Rev. 45, 1831 (1934).

${ }^{2}$ W. H. Bennett, Phys. Rev. 98, 1584 (1955).

${ }^{3}$ S. E. Graybill and S. V. Nablo, Appl. Phys. Lett. 8, 18 (1966).

${ }^{4}$ See National Technical Information Service Document No. ADA193185 (Naval Research Laboratory Memorandum Report No. 6159 by M. Lampe, 1988). Copies may be obtained from the National Technical Information Service, Springfield, Virginia 22161. The price is $\$ 17,00$ plus a $\$ 3.00$ handling fee. All orders must be prepaid.

${ }^{5}$ D. P. Murphy, M. Raleigh, R. A. Pechacek, and J. R. Greig, Phys. Fluids 30, 232 (1987).

${ }^{6}$ T. A. Peyser, J. A. Antoniades, R. F. Hubbard, M. C. Myers, D. P. Murphy, R. A. Pechachek, and R. A. Meger, Bull. Am. Phys. Soc. 36, 2379 (1991).

${ }^{7}$ S. S. Yu and R. E. Melendez, Bull. Am. Phys. Soc. 27, 1132 (1982).

${ }^{8}$ S. S. Yu, Bull. Am. Phys. Soc. 31, 890 (1986).

${ }^{9}$ C. L. Olson and J. W. Poukey, Phys. Rev. A 9, 2631 (1974); C. L. Olson, ibid. 11, 288 (1975).

${ }^{10}$ G. J. Budker, Sov. J. At. Energy 1, 673 (1956).

${ }^{\prime}$ R. J. Briggs, J. C. Clark, T. J. Fessenden, R. E. Hester, and E. J. Lauer, in Proceedings of the 2nd International Conference on High Power Electron and Ion Beam Research and Technology (Cornell U.P., Ithaca, NY, 1977), p. 319.

${ }^{12}$ M. A. Wilson, IEEE Trans. Plasma Sci. PS-28, 3375 (1981).

${ }^{13}$ W. E. Martin, G. J. Caporaso, W. M. Fawley, D. Prosnitz, and A. G. Cole, Phys. Rev. Lett. 54, 685 (1985).

${ }^{14}$ J. P. Smith, R. F. Schnieder, M. J. Rhee, H. S. Uhm, and W. Namkung, J. Appl. Phys. 60, 4119 (1986).

${ }^{15}$ S. L. Shope, C. A. Frost, G. T. Liefeste, C. E. Crist, P. D. Kiekel, J. W. Poukey, and B. B. Godfrey, IEEE Trans. Plasma Sci. PS-32, 3092 (1985).

${ }^{16}$ R. L. Carlson, S. W. Downey, and D. C. Moir, J. Appl. Phys. 61, 12 (1986).

${ }^{17}$ R. F. Lucey, R. M. Gilgenbach, J. D. Miller, J. E. Tucker, and B. A. Bosch, Phys. Fluids B 1, 430 (1989); R. F. Lucey, Ph.D. dissertation, University of Michigan, Ann Arbor, MI, 1988.

${ }^{18}$ K. J. O'Brien, G. W. Kamin, T. R. Lockner, J. S. Wagner, I. R. Shokair, P. D. Kiekel, I. Molina, D. J. Armistead, S. Hogeland, E. T. Powell, and R. J. Lipinski, Phys. Rev. Lett. 60, 1278 (1988).

${ }^{19} \mathrm{~J}$. D. Miller, IEEE Trans. Plasma Sci. PS-18, 658 (1990); Ph.D. dissertation, University of Michigan, Ann Arbor, MI, 1989.

${ }^{20}$ H. L. Buclaanan, Phys. Fluids 30, 221 (1987).

${ }^{21}$ R. C. Davidson, Physics of Nonneutral Plasmas (Addison-Wesley, Redwood City, CA, 1990), Chap. 4 and references cited therein.

${ }^{22}$ R. F. Hubbard, G. Joyce, S. P. Slinker, J. Krall, and M. Lampe, in Proceedings of the 7th International Conference on High Power Particle Beams, Karlsruhe, Germany, 1988 (Kernforschungszentrum Karlsruhe GMBH, Karlsruhe, 1988), p. 906.

${ }^{23}$ G. Joyce, R. Hubbard, and M. Lampe, in Ref. 22, p. 966.

${ }^{24}$ See National Technical Information Service Document No. DE89016670 (Sandia National Laboratory Report No. SAND87-2015, edited by J. Wagner and B. B. Godfrey, 1988). Copies may be obtained from the National Technical Information Service, Springfield, Virginia 22161. The price is $\$ 17.00$ plus a $\$ 3.00$ handling fee. All orders must be prepaid.

${ }^{25} \mathrm{D}$. R. Welch (private communication).

${ }^{26}$ E. P. Lee and R. K. Conper, Part. Accel. 7, 83 (1976).

${ }^{27}$ N. A. Krall and A. W. Trivelpiece, Principles of Plasma Physics (San Francisco Press, San Francisco, CA, 1986), pp. 361-364.

${ }^{28} \mathrm{~S}$. B. Swanekamp, Ph.D. dissertation, University of Michigan, Ann Arbor, MI, 1990.

${ }^{29}$ H. Alfvén, Phys. Rev. 55, 425 (1939).

${ }^{30}$ G. D. Warren, L. Ludeking, J. McDonald, K. Nguyen, and B. Goplen (private communication).

"J. Krall, K. Nguyen, and G. Joyce, Phys. Fluids B 1, 2099 (1989).

${ }^{32} \mathrm{~J}$. D. Lawson, in High Brightness Accelerators, edited by A. K. Hyder, M. F. Rose, and A. H. Guenther, (Plenum, New York, 1988), p. 5.

${ }^{33}$ R. C. Davidson, Theory of Nonneutral Plasmas (Benjamin, Reading, MA, 1975).

${ }^{34}$ H. S. Uhm, J. Appl. Phys. 56, 2041 (1984).

${ }^{35}$ J. D. Jackson, Classical Electrodynamics (Wiley, New York, 1975), Chap. 13. 